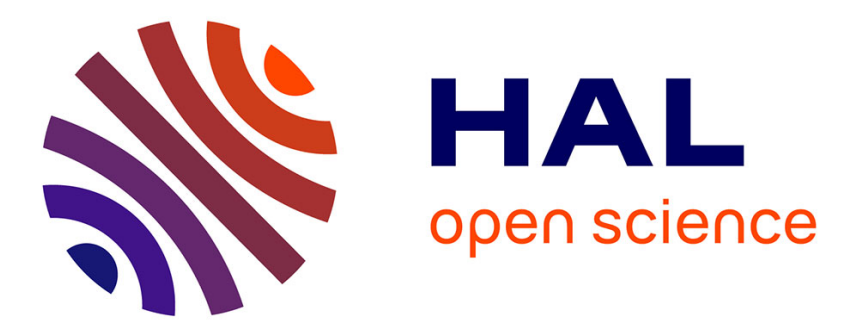

\title{
Full-field strain measurement and identification of composites moduli at high strain rate with the Virtual Fields Method
}

Raphael Moulart, Fabrice Pierron, Stephen R. Hallett, Michael R. Wisnom

\section{- To cite this version:}

Raphael Moulart, Fabrice Pierron, Stephen R. Hallett, Michael R. Wisnom. Full-field strain measurement and identification of composites moduli at high strain rate with the Virtual Fields Method. Experimental Mechanics, 2011, 51 (4), pp.509-536. 10.1007/s11340-010-9433-4 . hal-01064631

\section{HAL Id: hal-01064631 https://hal.science/hal-01064631}

Submitted on 16 Sep 2014

HAL is a multi-disciplinary open access archive for the deposit and dissemination of scientific research documents, whether they are published or not. The documents may come from teaching and research institutions in France or abroad, or from public or private research centers.
L'archive ouverte pluridisciplinaire HAL, est destinée au dépôt et à la diffusion de documents scientifiques de niveau recherche, publiés ou non, émanant des établissements d'enseignement et de recherche français ou étrangers, des laboratoires publics ou privés. 


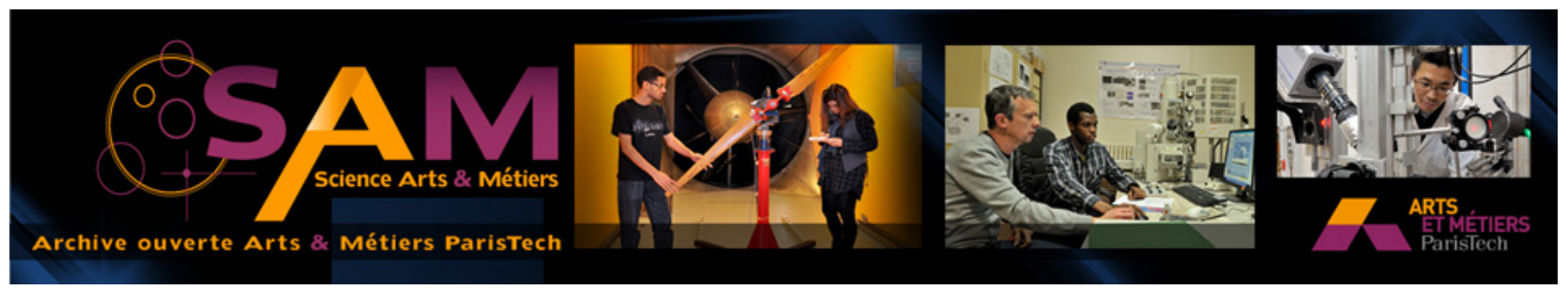

\section{Science Arts \& Métiers (SAM)}

is an open access repository that collects the work of Arts et Métiers ParisTech researchers and makes it freely available over the web where possible.

This is an author-deposited version published in: http://sam.ensam.eu

Handle ID: .http://hdl.handle.net/10985/8531

\section{To cite this version :}

Raphael MOULART, Fabrice PIERRON, Stephen R. HALLETT, Michael R. WISNOM - Full-field strain measurement and identification of composites moduli at high strain rate with the Virtual Fields Method - Experimental Mechanics - Vol. 51, n4, p.509-536 - 2011 


\title{
Full-Field Strain Measurement and Identification of Composites Moduli at High Strain Rate with the Virtual Fields Method
}

\author{
R. Moulart • F. Pierron • \\ S.R. Hallett · M.R. Wisnom
}

\begin{abstract}
The present paper deals with full-field strain measurement on glass/epoxy composite tensile specimens submitted to high strain rate loading through a split Hopkinson pressure bar (SHPB) device and with the identification of their mechanical properties. First, the adopted methodology is presented: the device, including an Ultra-High Speed camera, and the experimental procedure to obtain relevant displacement maps are described. The different full-field results including displacement, strain and acceleration maps for two mechanical tests are then addressed. The last part of the paper deals with an original procedure to identify stiffnesses on this dynamic case only using the actual strain and acceleration maps (without the applied force) by using the Virtual Fields Method. The results provide very promising values of Young's modulus and Poisson's ratio on a quasi-isotropic glassepoxy laminate. The load reconstructed from the mod-
\end{abstract}

R. Moulart $(\bowtie) \cdot$ F. Pierron (SEM member)

LMPF, Arts et Métiers ParisTech, Rue Saint Dominique

BP 508, 51006, Châlons-en-Champagne, France

e-mail: Raphael.Moulart@chalons.ensam.fr

F. Pierron

e-mail: Fabrice.Pierron@chalons.ensam.fr

S.R. Hallett · M.R. Wisnom

Department of Aerospace Engineering,

University of Bristol, Queen's Building,

University Walk, BS8 1TR, Bristol, UK

S.R. Hallett

e-mail: Stephen.Hallett@bristol.ac.uk

M.R. Wisnom

e-mail: M.Wisnom@bristol.ac.uk uli and strains compares favourably with that from the readings.

Keywords Full-field measurement • High strain rate • UHS camera • Grid method - SHPB • Virtual Fields Method · Fibre composites

\section{Introduction}

The identification of the mechanical behaviour of materials at high strain rates is still an open problem of great interest for the scientific community. One of the reasons is that at such strain rates, homogeneous stress and strain states are extremely difficult to obtain so that the usual identification procedures cannot be used accurately. Moreover, load measurement is also difficult in the early stages of the test because of inertia effects: the sample is not in quasi-static equilibrium.

This is the case for composite materials that are often used for aerospace applications where they are submitted to impacts, acceleration effects, etc. which are all dynamic loadings. In this context, it is important to be able to characterize the mechanical properties of such materials at high strain rates.

Due to the recent generalization of the use of full field kinematic measurements in combination with ultra-high speed imaging systems, the strain distribution in high-rate testing can be studied. Because of their apparent simplicity, these full-field methods are often thought to be "turnkey" solutions. However, to be relevant, such techniques have to be characterized in depth in terms of both resolution and spatial resolution in order to emphasize their potential but also their limitations. As part of this effort, the present work 
proposes an attempt to measure strain fields in tensile coupons loaded through a split Hopkinson pressure bar (SHPB) device. Full-field measurements are performed at rates up to 300,000 fps by using the grid method.

For a better understanding, the paper is divided into two main parts. The first one is devoted to the fullfield measurement of small strain using a Ultra-high speed camera. This part pays a particular attention to metrological issues concerning the measurements: indeed, they have to be good enough to lead to quantitative use to identify the stiffness components of the tested material. The second part introduces an attempt at such an identification, based on an original use of a well-known method. It leads to perfectible but very promising results. It should be noted that this paper is seminal in nature and provides ideas for future work more than ready-to-use solutions.

\section{State of the Art}

Full-Field Kinematic Measurements at High Strain Rates Using High-Speed Cameras

Historically, high-speed cameras have been used as imaging systems to give access to a visualization or a qualitative characterization of dynamic phenomena involved for instance in high strain rate testing. The idea to use them as full-field measurement techniques is newer and has been conditioned by the development of computer science and the generalization of digital images. Consequently, relatively few papers concerning full-field kinematic measurements in high strain rate testing are available in the literature. Some examples of such articles are given in this first section. This review is split in two main parts: the first one presents the studies involving high speed imaging systems (up to a few kfps for a field of view of 1 Mpixels), the second reports the investigations involving ultra-high speed systems (up to 1 Mfps for a field of view of 1 Mpixels).

Tiwari et al. studied the dynamic response of an aluminium sheet submitted to a buried blast loading [1]. To achieve this, they used 3D image correlation associated with a high-speed camera with interframe times between 16 and $40 \mu$ s. They measured surface velocities up to $220 \mathrm{~m} \cdot \mathrm{s}^{-1}$ and acceleration of $6 \cdot 10^{6} \mathrm{~m} \cdot \mathrm{s}^{-2}$ (600,000 g) during the first $30 \mu \mathrm{s}$. Reu and Miller also studied the deformation of an aluminium sheet under blast loading using 3D image correlation [2]. They underlined the gap that exists between highspeed imaging and ultra high-speed imaging systems and proposed to interlace two sets of two high-speed cameras to double the frame rate without affecting the spatial resolution. In a such a way, they managed to obtain displacement maps of $256 \times 256$ pixels with an interframe time of $20 \mu \mathrm{s}$ (frame rate of 50,000 fps). They concluded on the possible application of their method for frame rates going from 25 to $200 \mathrm{kfps}$. Tarigopula et al. used a SHPB device to load a high-strength dual phase steel [3]. They used an interframe time of $27 \mu \mathrm{s}$ (frame rate of $37,500 \mathrm{fps}$ ) which led to a field of view of $512 \times 128$ pixels. Using 2D image correlation, they managed to measure local strain rates from 150 to $600 \mathrm{~s}^{-1}$ and concluded on the early gradual transition from uniform strains to severely localized strains at these rates. Schmidt and Gilat also used a SHPB device to measure strains and strain rates in an aluminium sample submitted to a tensile loading [4]. They used stereo image correlation at a frame rate of $112,500 \mathrm{fps}$ and with a rather small field of view $(256 \times 48$ pixels $)$. They compared the average strains to the strains obtained from a strain gauge bonded onto the sample. They eventually concluded that "direct measurement of thousands of local strains is superior to inference of average strain based on assumptions of equilibrium and uniformity which do not always occur in practice".

Two very interesting papers dealing with first attempts at identification of mechanical properties from full-field measurement at high strain rates have to be quoted. In the first one, Kajberg and Wikman tried to identify visco-plastic parameters of a mild steel submitted to a compression loading through a SHPB set up [5]. They used a dog-bone shaped sample in order to get inhomogeneous strains from 2D image correlation measurements. They compared their fields to finite element (FE) analysis simulations using a JohnsonCook material model. They identified visco-plastic parameters through a least-square updating procedure on a cost function comparing experimental and FE data. Unfortunately, their results, obtained with an interframe time of $8 \mu \mathrm{s}$, present a very small field of view $(166 \times 192$ pixels $)$ which did not allow to completely describe the localization process due to lack of spatial resolution. The second paper by Avril et al. deals with the identification of elasto-visco-plastic constitutive parameters at more moderate strain rates (around $1 \mathrm{~s}^{-1}$ ) [6]. The authors used digital image correlation on dogbone steel specimens submitted to a tensile test on a universal hydraulic test machine. They used a time step of $200 \mu$ s. The heterogeneity of the strains allowed them to simultaneously identify all the parameters of the constitutive equation using the virtual fields method including a strain rate sensitivity as the strain rate maps were heterogeneous. They concluded that "extension 
to very high strain rates is one of the main prospects for this approach because the transient effects would be augmented. The recent drastic improvements in the technology of high-speed cameras will soon provide suitable conditions for these experiments."

All the previously quoted papers dealt with the use of mono-sensor high-speed cameras (single CMOS sensors technology). These cameras propose a field of view related to the frame rate: the higher the frame rate, the lower the field of view which leads to compromises that are not always satisfying in terms of both spatial and temporal resolutions. Especially, for a full spatial resolution (1 Mpixels), they only allow to reach a few kfps.

An alternative to these cameras are the multi-sensor ultra high-speed cameras. They allow to record images with a full resolution for a wide range of frame rates. Their main drawback is the fact that they record frames on different CCD or CMOS sensors which can generate systematic errors coming from intensity variations, small misalignments, etc. A recent paper by Tiwari et al. clearly underlines the problems encountered with such cameras and proposes a few solutions to try to improve the performances [7]. Another drawback for these types of cameras is that they only allow to record a constant limited number of frames (equal to the number of sensors) which limits the duration of the acquisition and thus requires a very accurate control of the triggering. Another UHS camera based on a mono-sensor technology with an embedded memory does exist (Shimadzu HPV-1 and HPV-2 that record 102 images with a minimum interframe time of $1 \mu \mathrm{s}$ ). Unfortunately, for the moment, the field of view of these cameras is rather limited, typically $312 \times 260$ pixels which is too low to perform full-field measurements with appreciable spatial resolution. More details about high speed imaging technologies can be found in [8].

A few papers mentioning the use of such ultrahigh speed imaging systems can be found in the literature. Luo et al. studied the compressive behaviour of silicon aerogels at high strain rates [9]. They used a SHPB device coupled with a Cordin 550-62 camera $(1,000 \times 1,000$ pixels $)$ with an interframe time acquisition of $24 \mu \mathrm{s}$ (frequency of 41,000 fps). To measure displacement and strain maps, digital image correlation was performed. They observed the failure behaviour of the aerogel and noticed that there was no significant localization of the compaction for a nominal compressive strain of $17 \%$ concluding that the failure mechanism was not the same as that observed for quasi-static loadings. They also calculated the evolution of Poisson's ratio from the ratio of the average measured longitudinal and transverse strains $(v=$ $-\overline{\varepsilon_{y y}} / \overline{\varepsilon_{x x}}$ ). In the same way, Luo et al. studied the compressive behaviour of vanadia aerogels using a SHPB and the same camera with an interframe time of $14 \mu \mathrm{s}$ (frequency: 72,000 fps) [10]. Once again, the strains appeared to be mainly homogeneous at these high-strain rates. Siviour studied the wave propagation in a rod of PMMA submitted to a compression loading performed thanks to a SHPB device by using a SIM16 ultra-high speed camera $(1280 \times 960$ pixels $)$ coupled with digital image correlation (DIC) [11]. The interframe time was $2.5 \mu$ s (frequency: 400,000 fps), the exposure time, $0.5 \mu \mathrm{s}$. The author compared the displacement obtained by DIC for a reference point of the sample with the one obtained by integrating the strain curves of the input bar and concluded on their good agreement.

In the three last quoted papers, ultra-high speed imaging systems have been used to measure full-field of rather large strains (several tens of percent) $[9,10]$ or to extract a single displacement value from the fullfield measurement (thus not really taking advantage of the available spatial information) [11]. A very recent paper also mentions the use of a Cordin 550-32 UHS camera to measure strain maps of small magnitude [12]. In this paper, the performances of the camera have been investigated in depth (through a study of the image intensity variability, translation and rotation tests). Then, a three-point bending test was performed on a cracked syntactic foam coupon thanks to a drop weight machine. The aim was to follow the growth of the crack and determine the fracture parameters of the tested material. To reach this purpose, digital image correlation was applied on pictures acquired with an interframe time of $5 \mu$ s (frequency: 200,000 fps). The procedure developed to compute the displacement maps was of the same kind as the one proposed here. The authors managed to obtained relevant strain maps with a good resolution (around $10^{-4}$ ) that were in accordance with the results given by a finite element simulation.

In the present study, and in the same way as in [12], it is proposed to use such a camera to acquire kinematic fields during a high strain rate test. This is applied to a composite coupon submitted to a tensile loading through a SHPB device. In these conditions, before the occurrence of the global failure of the sample, the strains are rather low (inferior to $1 \%$ ) which is challenging for such full-field measurements.

To complete this short review on full-field kinematic measurements applied to high strain rate testing, the next section gives a short overview of the literature concerning the dynamic behaviour of composite materials. 
Dynamic Behaviour of Laminated Composites

As mentioned in "Introduction", high strain rate testing of composite materials is an important topic since many of their applications result in dynamic loading. It is therefore necessary to characterize any material property rate effect in order to ensure accurate and conservative design. To this end there have been numerous studies aimed at such high rate characterization, much of this has involved adaptations of the well known split Hopkinson or Kolsky bar. Early work on developing a tensile testing technique for composites was undertaken by Harding and Welsh [13]. The bar design previously used for metals consisted of a weighbar tube surrounding the specimen and inertia bar which impacted a yoke at the end of the apparatus thereby causing a tensile wave to propagate through the specimen [14]. The composite specimens were glued into slots in the bars which caused some difficulty with obtaining a gauge section failure rather than shear failure of the adhesive. Also the specimens were significantly longer than had been traditionally used for metals in order to minimize stress concentrations. Woven glass fibre reinforced epoxy specimens were shown to have more than two fold increase in modulus and strength with an increase in strain rate from $10^{-4} \mathrm{~s}^{-1}$ to $870 \mathrm{~s}^{-1}$. Staab and Gilat found similar strain rate effects for angle ply glass/epoxy specimens [15]. These were tested using an alternative form of tensile Hopkinson bar in which the input bar was clamped and then preloaded [16]. The stress wave is then achieved by rapid release through failure of an aluminium pin. The design of tensile Hopkinson bar apparatus used in the present work follows that of $\mathrm{Li}$ et al. [17] in which a tubular projectile sits around the input bar although here this is fired down a gun barrel by pressurized gas instead of mechanical loading as in [17].

There have been many other high strain rate characterizations of composite materials using various adaptations of Hopkinson bar and drop weight impactors since these early experimental results $[18,19]$. The general trend is for increasing stiffness with increasing strain rate, particularly in the case of glass fibre composites e.g. [20,21]. Carbon fibre composites are generally less strain rate sensitive in the fibre direction [13] but in offaxis [22] or through thickness directions [23] the strain rate sensitivity of the matrix can have some influence. It can thus be seen that there is clearly a need for accurate high rate characterization of composite materials. The full-field measurements combined with the virtual fields method proposed here provide a novel method for determining material properties through direct measurement from the specimen, thus avoiding many of the experimental difficulties encountered in previous works.

Notch sensitivity of composites is also a significant field of study owing to the importance of this topic for the design of composite components [24]. Despite the well documented effect of strain rate on composite material properties there has been very little documented work on high strain rate testing of notched composites. This is perhaps in part due to the requirement to use small specimen geometries to achieve suitably high strain rates. Strain rates of up to $102 \mathrm{~s}^{-1}$ have been achieved in a servo hydraulic machine by Kazemahvazi et al. [25] who observed that for noncrimp glass fibre/vinyl-ester notch sensitivity is not strongly affected by strain rate for laminates with a high proportion of $0^{\circ}$ plies but decreased for those with $50 \% 0^{\circ}$ plies. Chen and Hallett [26] have tested quasi-isotropic carbon/epoxy open hole tension specimens up to $500 \mathrm{~s}^{-1}$ using the same Hopkinson bar as presented here. Results show that depending on the layup notched strength can either increase or decrease as a result of the complex way in which the damage develops. Owing to the complexity of such specimens over and above more traditional high rate tests there is significant benefit in being able to accurately measure the strain field over the duration of the test such as has been achieved here.

\section{Part I: Small Strain Measurements Using an Ultra High Speed Camera and the Grid Method}

Methodology

\section{Experimental setup}

The experimental setup consisted in a SHPB device designed to apply a tensile load on a specimen located between the input and the output bars. To perform the dynamic loading, a projectile was shot onto the input bar, creating a strain wave that was partially transmitted through the sample (Fig. 1). The bars are cylindrical, $1.6 \mathrm{~m}$ long, $16 \mathrm{~mm}$ wide. The input bar is screwed onto a loading bar also cylindrical, $2.8 \mathrm{~m}$ long, $32 \mathrm{~mm}$ wide. The projectile is cylindrical, $400 \mathrm{~mm}$ long with an interior diameter of $41.75 \mathrm{~mm}$ and an exterior diameter of $63.5 \mathrm{~mm}$. To perform the loading, it hits a shoulder put at the end of the loading bar. With this kind of device, strain rates up to $10^{3} \mathrm{~s}^{-1}$ can typically be obtained [19]. A review of the use of SHPB can be found in [27].

The tested specimens were $50 \mathrm{~mm}$ long, $16 \mathrm{~mm}$ wide quasi-isotropic laminated composite coupons manufac- 
Fig. 1 The split Hopkinson pressure bar device (dimensions in $\mathrm{mm}$ )
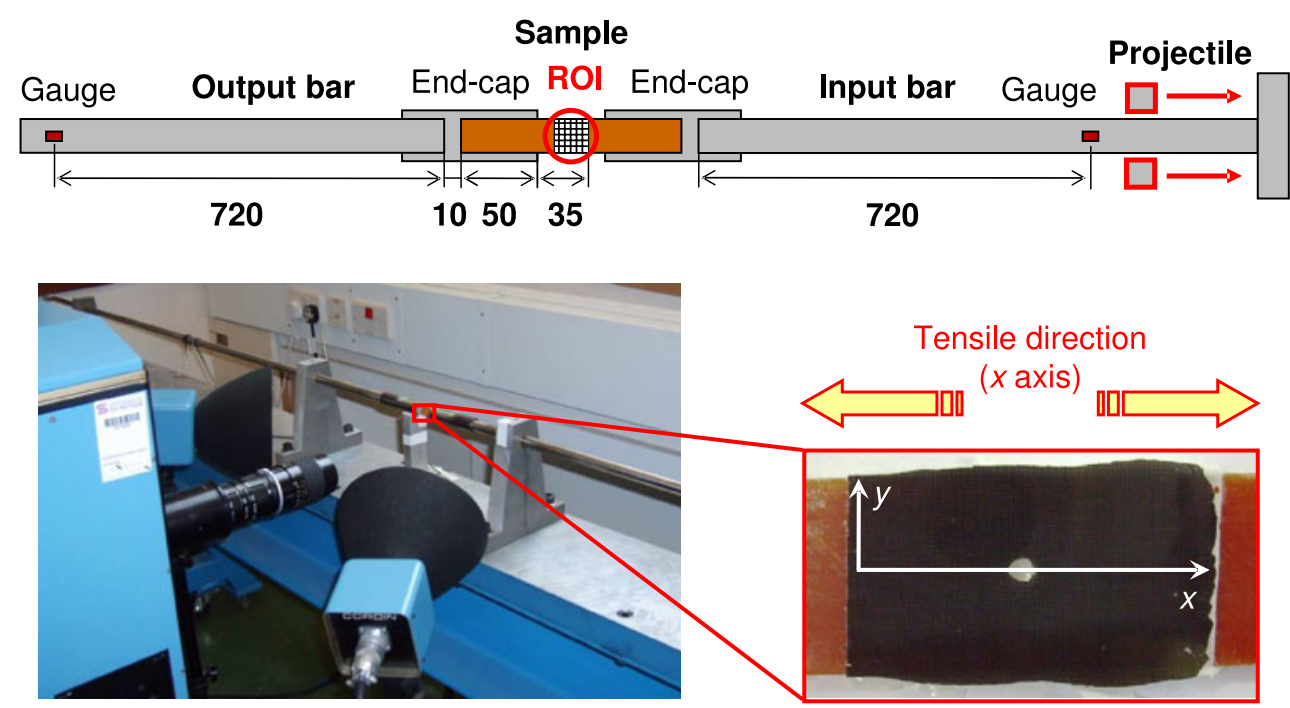

tured from pre-impregnated E-glass and 913 epoxy. The first one was $2 \mathrm{~mm}$ thick with a $\left[45_{2} / 90_{2} /-45_{2} / 0_{2}\right]_{\mathrm{s}}$ layup and presented a $3.2 \mathrm{~mm}$ diameter open circular hole. The second one was $1 \mathrm{~mm}$ thick with a [45/90/$45 / 0]_{\text {s }}$ layup and did not present any hole. On these specimens, a cross-grid of $200 \mu \mathrm{m}$ pitch ( 5 lines per $\mathrm{mm}$ ) was bonded. It was obtained from a set of cross lines (printed on a polymeric film thanks to a high resolution printer) bonded on the sample using an epoxy glue. To ensure good contrast, the chosen glue was white [28]. This grid constituted a periodic pattern that has been processed by the so-called grid method. This method determined the phase of this periodic pattern using a spatial phase shifting algorithm. The difference of phases between an initial and an actual state is directly proportional to the displacement within the specimen in a considered direction. With this method, one dis- placement value per period of the grid is obtained. Consequently, its spatial resolution is equal to the pitch of the grid. More details about this full-field measurement method can be found in the following references: $[29,30]$.

To record the pictures of the grid during the mechanical test, an ultra-high speed camera, the Cordin 550-62 coupled with a Cordin model 659 flash light source (Energy: 1,110 J; duration: from 0.5 to $11 \mathrm{~ms}$ ) were used. The camera is based on a rotating mirror that directs the light successively to 62 multiplexed CCD sensors. The camera records the different states of loading on these different sensors. The frame rate of recording is given by the rotation speed of the mirror (Fig. 2). With this device, a minimum interframe time of $0.25 \mu$ s can be reached (i.e., a maximum frequency of acquisition of 4,000,000 fps). In this study, due to light
Fig. 2 Principle of the multi-sensor Ultra High Speed (UHS) camera

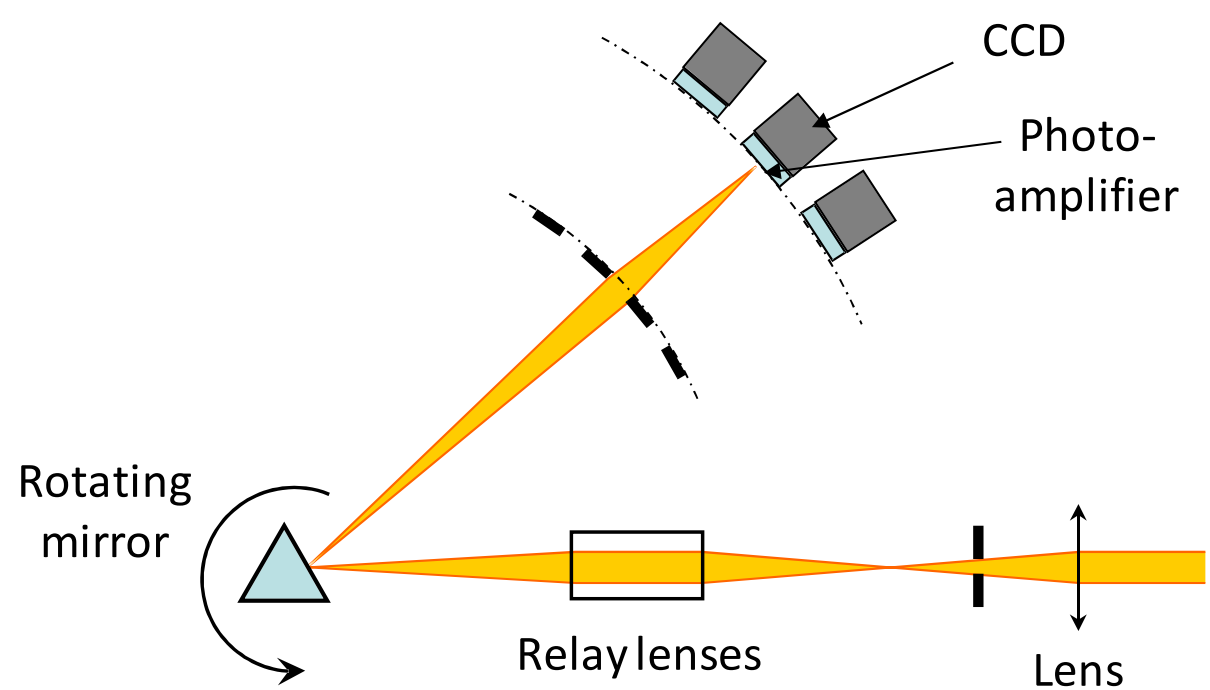


and triggering issues, the interframe time was limited to $3.3 \mu$ s (i.e., a frequency of acquisition of $300,000 \mathrm{fps}$ ).

Considering the size of each sensor $(1,000 \times 1,000$ pixels), the size of the observed region, the pitch $(200 \mu \mathrm{m})$ and the magnification, the sampling of the grid was 9 pixels per period for the first test and 11 pixels per period for the second one.

The camera that was used in the present study originates from the EPSRC Engineering Instrument Pool. More details about the type of UHS camera used here can be found in $[8,31]$.

\section{Noise study-experimental procedure}

To determine the accuracy of the full-field measurement method, it was necessary to perform a preliminary study to quantify its resolution i.e., the smallest significant displacement value that can be measured. For this purpose, a definition of this displacement resolution has to be given. Assuming that the possible errors were only due to a spatially independent white noise coming from the acquisition system, the displacement resolution was defined as the standard deviation of this noise. This has been easily measured by capturing pictures of the same region of interest of the still sample (without any loading). The displacement between these two nominally identical states should be uniformly null. But, in fact, due to the digitization noise, it was not. The standard deviation of this "noise map" is the displacement resolution of the method.

By calculating the displacement incrementally from one sensor of the camera to another, there would have been a spatially correlated bias adding up to the white noise previously mentioned. This is due to the fact that from one sensor to another, there can be small misalignments, slight rotations and variations of the light intensity that are inducing non negligible errors.

The proposed solution to obtain relevant displacement maps is to take a first set of 62 still images of the grid from each CCD sensor and a second set of actual deformed pictures during the test. The displacement is obtained sensor by sensor by difference between the actual and the initial sets of pictures as explained in [32].

In this configuration, "noise maps" are showing a distribution close to a spatially independent white noise (Fig. 3). However, there is still a bias (especially, the mean values of "noise maps" are not centered on zero).
Fig. 3 Improved noise maps obtained by calculation sensor by sensor from two different sets of pictures (colormap in $\mu \mathrm{m}, 1$ period of the grid $\equiv 200 \mu \mathrm{m} \equiv 9$ pixels; field of view: $20 \times 16 \mathrm{~mm}$ )
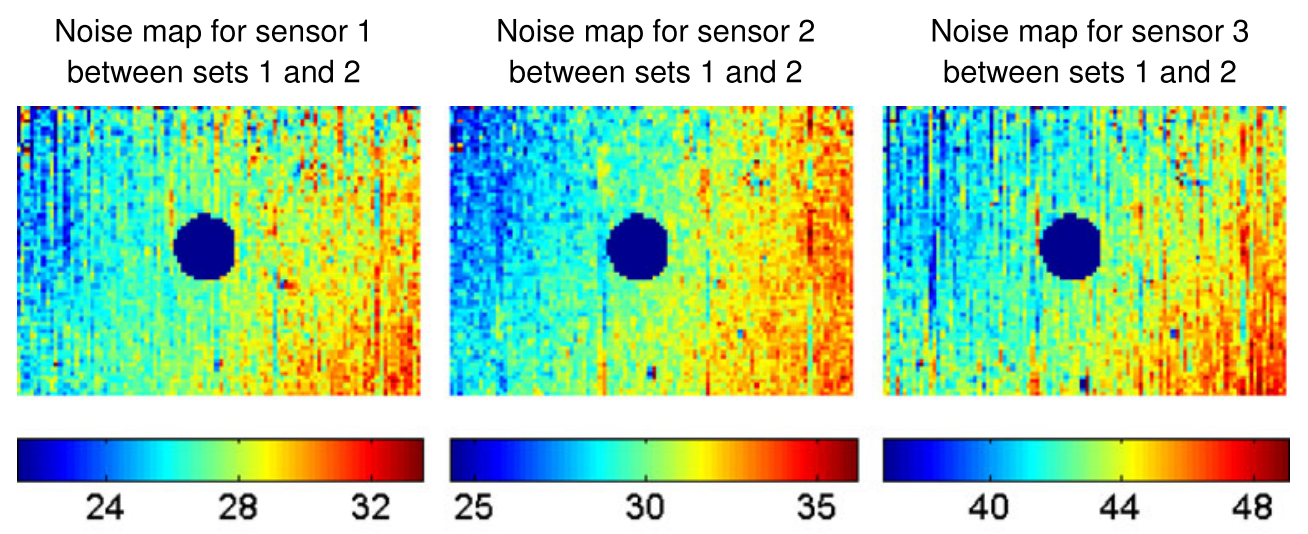

Noise map for sensor 5

between sets 1 and 2
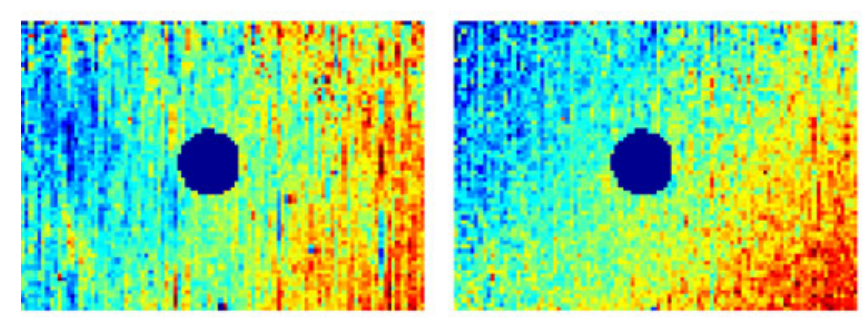

Noise map for sensor 6

between sets 1 and 2
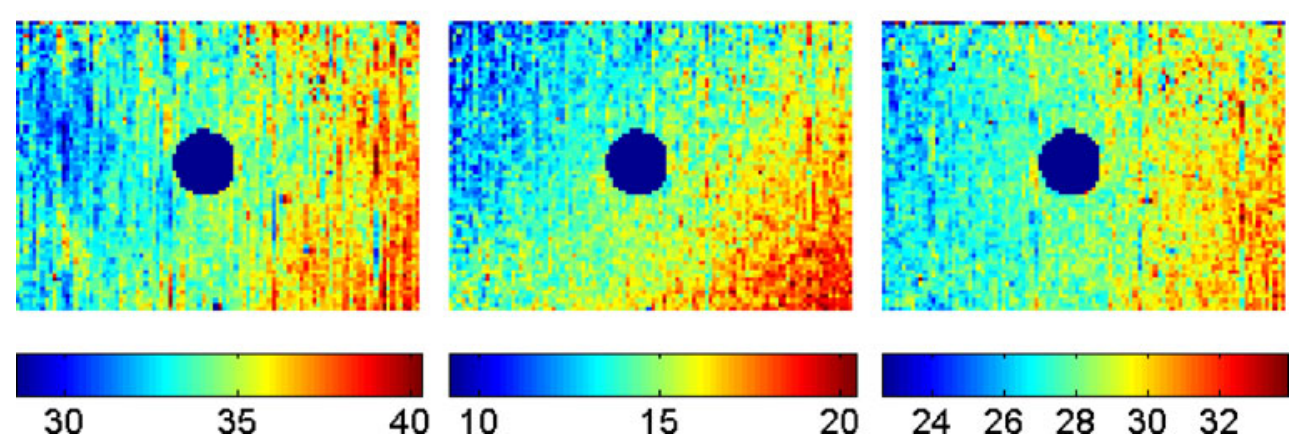

10

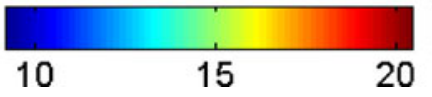

$\begin{array}{lllll}24 & 26 & 28 & 30 & 32\end{array}$ 
This bias could be due to some vibrations generated by the rotation of the mirror in the camera that could induce slight movements of the sensors from their initial positions. It has to be noted that the shift of the average value does not affect strains (as strains are displacement gradients) but does affect the acceleration.

A graph of the standard deviation and average values of the noise maps for each sensor is plotted on Fig. 4. It shows quite large variations from one sensor to another in terms of standard deviation due especially to the variation of light intensity from one sensor to another. Nevertheless, from this curve, it can be seen that the standard deviation of noise maps is between 0.5 and $1.8 \%$ of the pitch i.e., between 1 and $3.6 \mu \mathrm{m}$ for a pitch of $200 \mu \mathrm{m}$ (for a static test, one can typically obtain a displacement resolution between 0.3 and $0.5 \%$ of the pitch $[33,34])$. It has to be noted that on this curve, there are two missing points. Indeed, the Cordin camera records 64 frames but possesses only 62 physical CCD sensors. The two missing sensors have been removed to allow the light to pass; they correspond to blank pictures in the final set.

This value of displacement resolution can be compared with that obtained by Kirugulige et al. with the same UHS camera [32]. By applying Digital Image
Correlation to pictures obtained from the different sensors of the camera, they obtain a displacement resolution between 0.02 and 0.08 pixel (with a size of $32 \times 32$ pixels for the subset image on which the digital image correlation is applied). The values of 0.5 and $1.8 \%$ of the pitch that are obtained here can be translated respectively as 0.05 and 0.16 pixels which is approximately twice what is obtained by Kirugulige et al. Nevertheless, in the present study, the spatial resolution (equal to the period of the grid) is much better: as one pitch was sampled by 9 pixels, it leads to an equivalent size of subset image of $9 \times 9$ pixels that is to say an area 13 times smaller than the one used by Kirugulige et al. As the averaging of the displacement on a square of $P \times P$ pixels divides the standard deviation of the noise by $\sqrt{P^{2}}=P$, the resolution for identical spatial resolution is between $0.05 / \sqrt{13}$ and $0.16 / \sqrt{13}$ that is to say between 0.01 and 0.05 pixel. So, it can be concluded that the results obtained here are slightly better than in [32] at the cost of bonding a grid.

This approach to compute displacements (sensor by sensor from different recording sets) was used in the rest of this work. It has to be underlined that with this solution, and considering the nature of the measurand used to obtain the displacement (a phase), phase
Fig. 4 Level of noise plotted for each sensor ( 1 period of the grid $\equiv 200 \mu \mathrm{m} \equiv 9$ pixels $)$
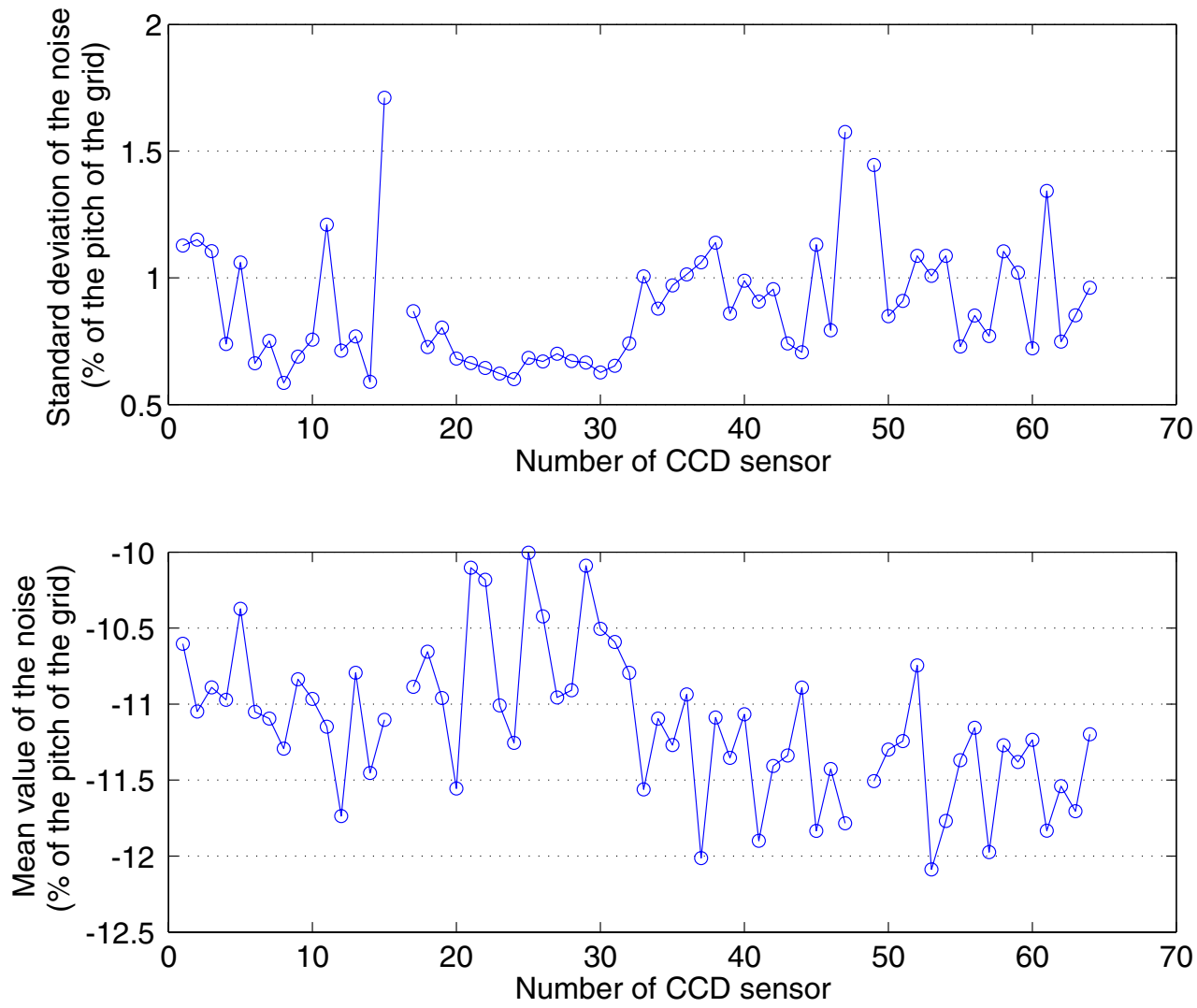
wrapping necessarily occurs. This is to say that the value of the phase of the signal within a single frame is known modulo $2 \pi$ (leading to knowledge of the displacement modulo $p$ ). A phase unwrapping algorithm had to be used to fix this problem but is not explained since it is not the aim of the present paper. More details about it can be found in [35]. To ensure that the absolute value of displacement obtained after phase unwrapping is the right one (as it could be affected by a constant value equal to $k \times p$ ), each displacement map has been checked manually using the hole or some grid defect as the feature to follow.

To pursue the noise study, the strain resolution has been calculated. To determine the strain maps from the displacement maps, the latter have to be smoothed before being numerically differentiated to reduce the effect of the noise (which is amplified by differentiation). To this purpose, a diffuse approximation algorithm has been used. This algorithm is based on a local polynomial fitting with a particular weighting function. More details about it can be found in [36, 37]. This method is based on a local polynomial regression (using a local weighted least square algorithm) that gives smoothed reconstructed displacement fields. The main parameter of this algorithm is the "critical radius" that determines the span of the window of influence for the reconstruction at each point and thus the spatial resolution of the strain maps. By applying this algorithm with several radii to the "noise maps", the curves of standard deviations of "strain noise maps" shown on Fig. 5 have been obtained. They confirm that the larger the radius, the lower the noise and, consequently, the better the strain resolution. By applying a radius equal to 10 periods, the standard deviation is about $10^{-3}$.
This is consistent with the order of magnitude of strain that had to be measured in this study. Consequently, this radius has been used in the rest of this work as it seems to be a good compromise between spatial and strain resolutions. Moreover, it will be seen in "Part II: Identification of Mechanical Properties Using the Virtual Fields Method" that the choice of the radius of the diffuse approximation does not really affect the identification of the mechanical properties with the proposed approach.

In the same way, strain rate and acceleration resolutions have been quantified. To compute the strain rate, a simple finite differences algorithm has been used:

$\dot{\varepsilon}_{i j}(t)=\frac{\varepsilon_{i j}(t)-\varepsilon_{i j}(t-1)}{\Delta t}, i=(x, y), j=(x, y)$.

The curves obtained from the standard deviations of "noise strain rate maps" (Fig. 6) show that the strain rate resolution was approximately between 100 and $300 \mathrm{~s}^{-1}$.

To compute the acceleration by double temporal differentiation, the smoothed displacement maps reconstructed by diffuse approximation are used. The acceleration have firstly been obtained thanks to a simple finite differences algorithm:

$a_{i}(t)=\frac{U_{i}(t+1)+U_{i}(t-1)-2 \times U_{i}(t)}{\Delta t^{2}}, i=(x, y)$.

In terms of noise, this leads to the curves plotted on Fig. 7. They show a standard deviation up to $2 \times 10^{5} \mathrm{~m} \cdot \mathrm{s}^{-2}$ but, above all, an average value going from $-2 \times 10^{6}$ to $2 \times 10^{6} \mathrm{~m} \cdot \mathrm{s}^{-2}$. This shift of the
Fig. 5 Level of strain noise plotted for each sensor

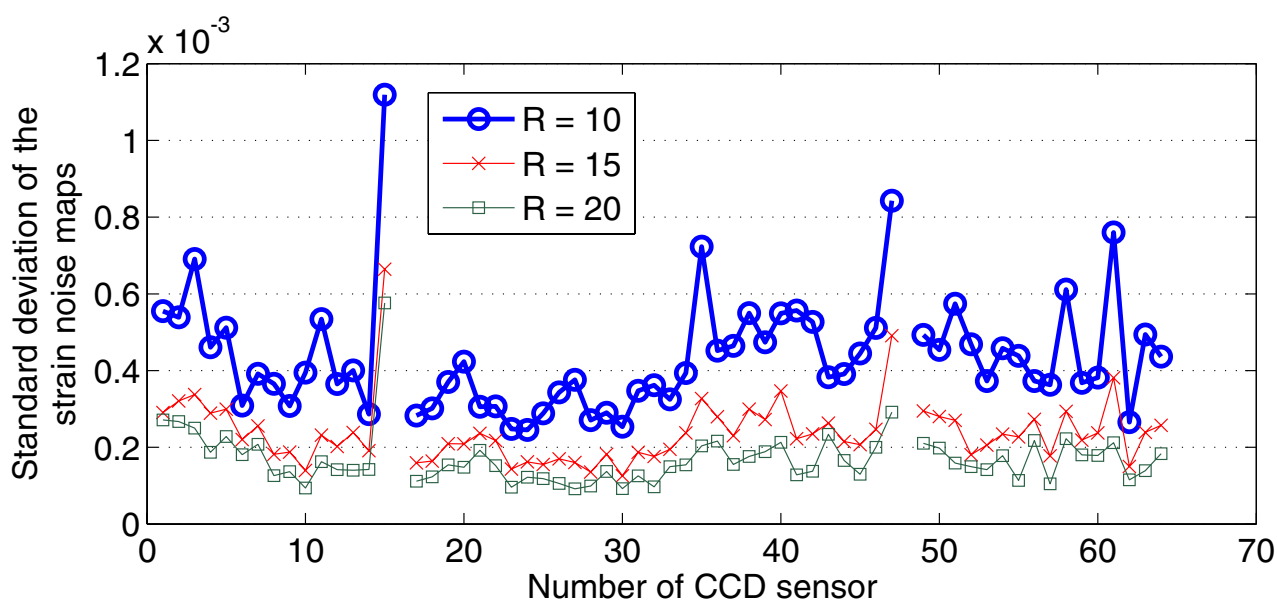


Fig. 6 Level of strain rate noise plotted for each sensor

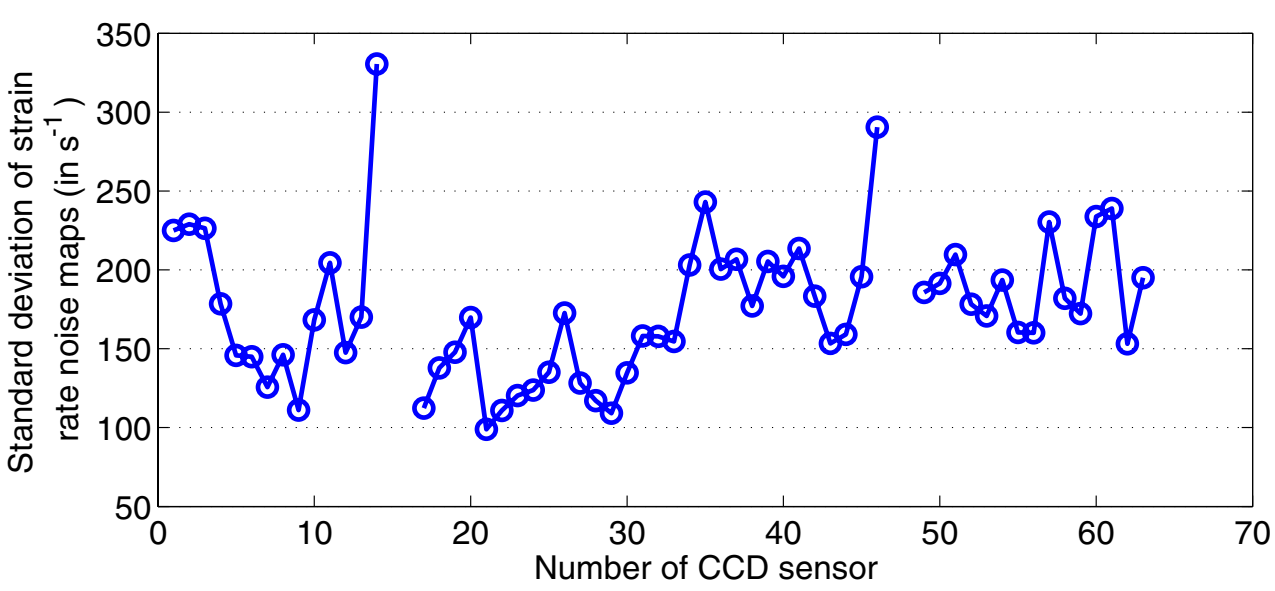

average value of the acceleration maps comes from the systematic bias that affects displacement maps. This kind of results in terms of acceleration is not compatible with the order of magnitude of what has been measured here $\left(\simeq 10^{6} \mathrm{~m} \cdot \mathrm{s}^{-2}\right)$. Consequently, a temporal smoothing has been applied to the results (in addition to the spatial one) to allow to get relevant acceleration maps. This smoothing was performed as follows: for every pixel, the displacement at a given stage is assumed to be a fourth order polynomial function of time. To reconstruct it, a local least square algorithm applied on a sliding window of nine images has been
Fig. 7 Level of acceleration noise reconstructed by finite differences plotted for each sensor
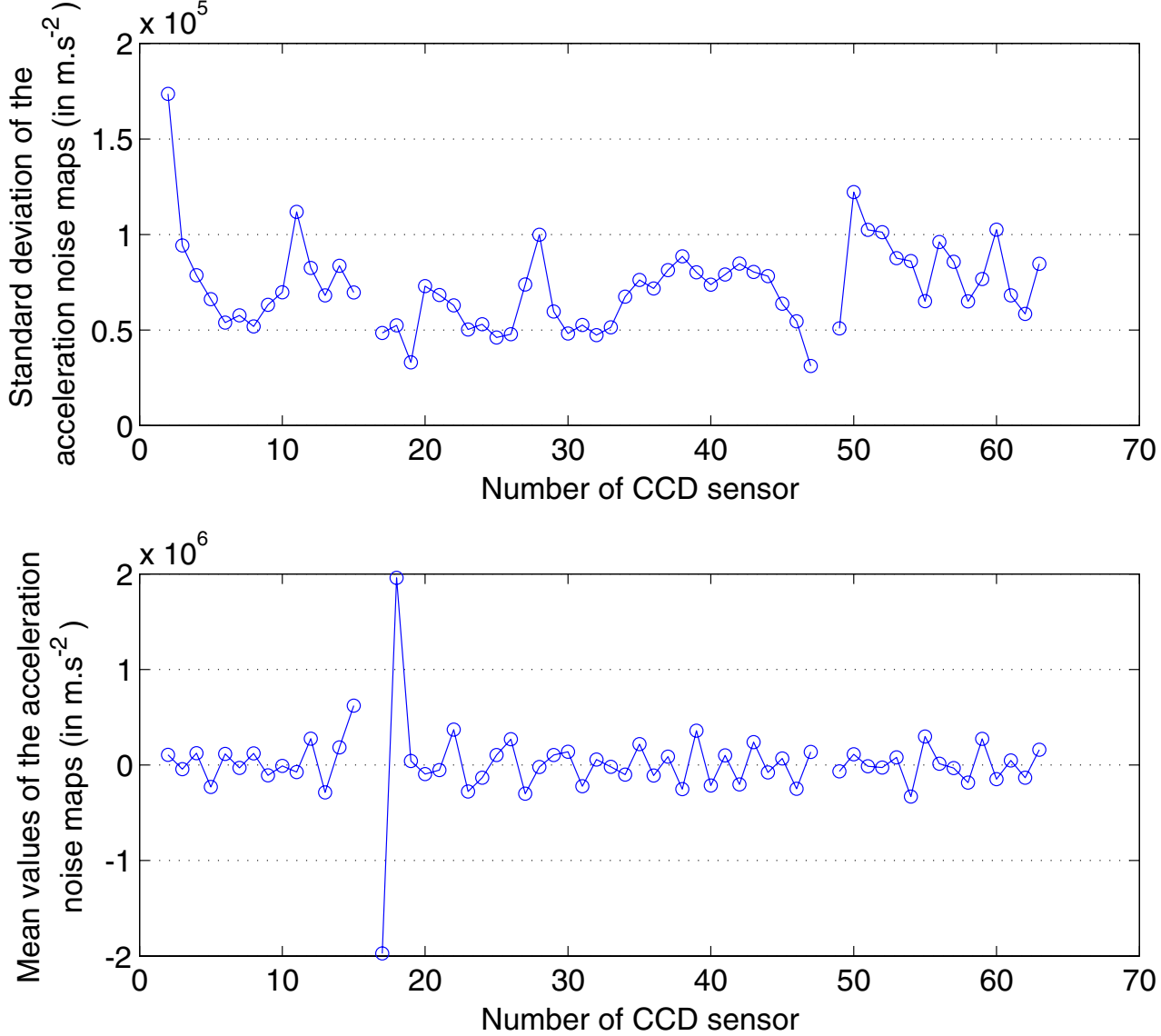
applied. Consequently, the acceleration was a second order polynomial function of time obtained by double differentiation of the reconstructed displacements. By applying this to the "noise maps", results shown on Fig. 8 have been obtained. They show a global reduction of the level of noise affecting acceleration maps. Especially, with this approach, the average of "noise acceleration" is contained between $-2 \times 10^{5}$ and $4 \times$ $10^{5} \mathrm{~m} \cdot \mathrm{s}^{-2}$. Thus, this solution has been adopted to compute accelerations. However, this large remaining bias on acceleration has to be taken into account. It will be shown in "Part II: Identification of Mechanical Properties Using the Virtual Fields Method" how this can disturb the identification of the mechanical properties.

\section{Results}

In this section, two sets of results are presented. The first one corresponds to the test performed on an openhole tensile coupon and the second one corresponds to the test performed on a coupon without hole. It has to be noted that for the first test, no loading has been recorded.

Both tests have been performed using the SHPB device. The velocity of the projectile at the moment of the impact was about $25 \mathrm{~m} \cdot \mathrm{s}^{-1}$ for the first test and $23 \mathrm{~m} \cdot \mathrm{s}^{-1}$ for the second one, values which have achieved average strain rates of approximately $500 \mathrm{~s}^{-1}$ in other tests [26].

\section{First test: open-hole tensile coupon}

A set of 62 frames has been recorded with the camera at a frequency of $300,000 \mathrm{fps}$. On this set, a certain number of frames have been recorded before the strain wave passed through the sample. The time $t=0$ of this test has been considered to be the last frame recorded without any significant displacement, that is to say frame 35. On frame $44(t=30 \mu \mathrm{s})$, the first crack on the sample occurred; calculation of the full-field strain maps is meaningless then since a discontinuity that will disturb the calculation of full-field strains has appeared.
Fig. 8 Level of acceleration noise reconstructed by 4 th order polynomial fitting of the displacement plotted for each sensor
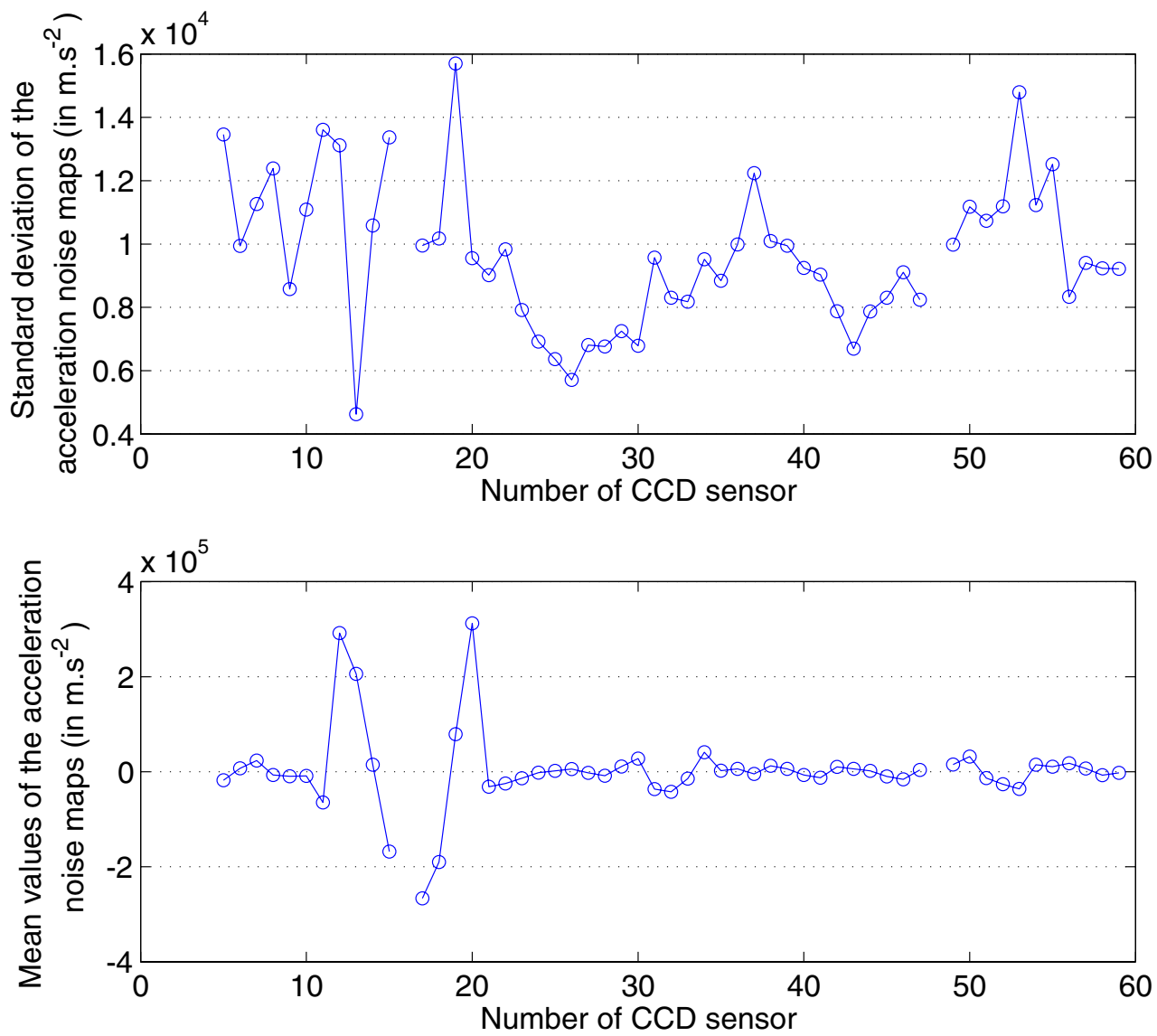
Therefore, only 8 meaningful strain maps have been obtained before the onset of surface cracking.

On Fig. 9, apparition and growth of cracks can be observed. They all present an angle of $45^{\circ}$ which is induced by the angle of the fibres in the surface ply. The two main cracks occur from the hole to the edge of the sample. It can be seen that the one below the hole appears and reaches the edge of the sample first. It can also be seen that the first cracks appearing after these two main ones are located at the bottom left of the sample. This seems to indicate that the tensile loading is not exactly symmetrical and is higher on the lower part of the sample.

Figure 10 shows the displacement maps plotted after removing the average value in order to avoid the scale effects induced by the global rigid body motion and to concentrate on their gradients within the sample (that is to say that Fig. 10 shows $U_{x}-\overline{U_{x}}$ maps, where $\overline{U_{x}}$ is the average value). The isodisplacement lines are tilted which shows that there might be some solid rotation but also in-plane bending.

The reconstructed $\varepsilon_{x x}$ maps are shown on Fig. 11. These strain maps clearly show that the value of the normal strain in the direction of the loading is higher in the lower part of the sample than in its upper part. This confirms what has been expected by looking at the spatial localization of the cracks and indicates that there are some in-plane bending effects superimposed onto the tensile loading. This is most likely due to the way the sample is fixed to the bars, since bending strains in the loading bars have been previously checked using back to back strain gauges. Indeed, the samples were glued to end-caps that were then screwed onto the bars (Fig. 12). The bending effects can have been induced by a slight misalignment of the sample in the end-caps or by the clearance in the screw attachments. In this case, the effect should not be reproducible: the bending effect should not occur with the same intensity and not necessarily in the same direction which can be observed on the second test where the longitudinal strain is higher on the upper part of the sample (see "Second test: tensile coupon without hole"). The strain value at the hole just before the crack appears $(26.7 \mu \mathrm{s})$ is about $1 \%$, which is consistent with results in [38] in static.

On the $\dot{\varepsilon}_{x x}$ maps deduced from the strains (Fig. 13), it can be observed that the strain rate at the vicinity of the hole is approximately $800 \mathrm{~s}^{-1}$ at the last stage of loading before the occurrence of the first crack.
Fig. 9 Evolution of the cracks on the intensity pictures (test 1 , tensile direction: horizontal, field of view: $20 \times 16 \mathrm{~mm}$ )
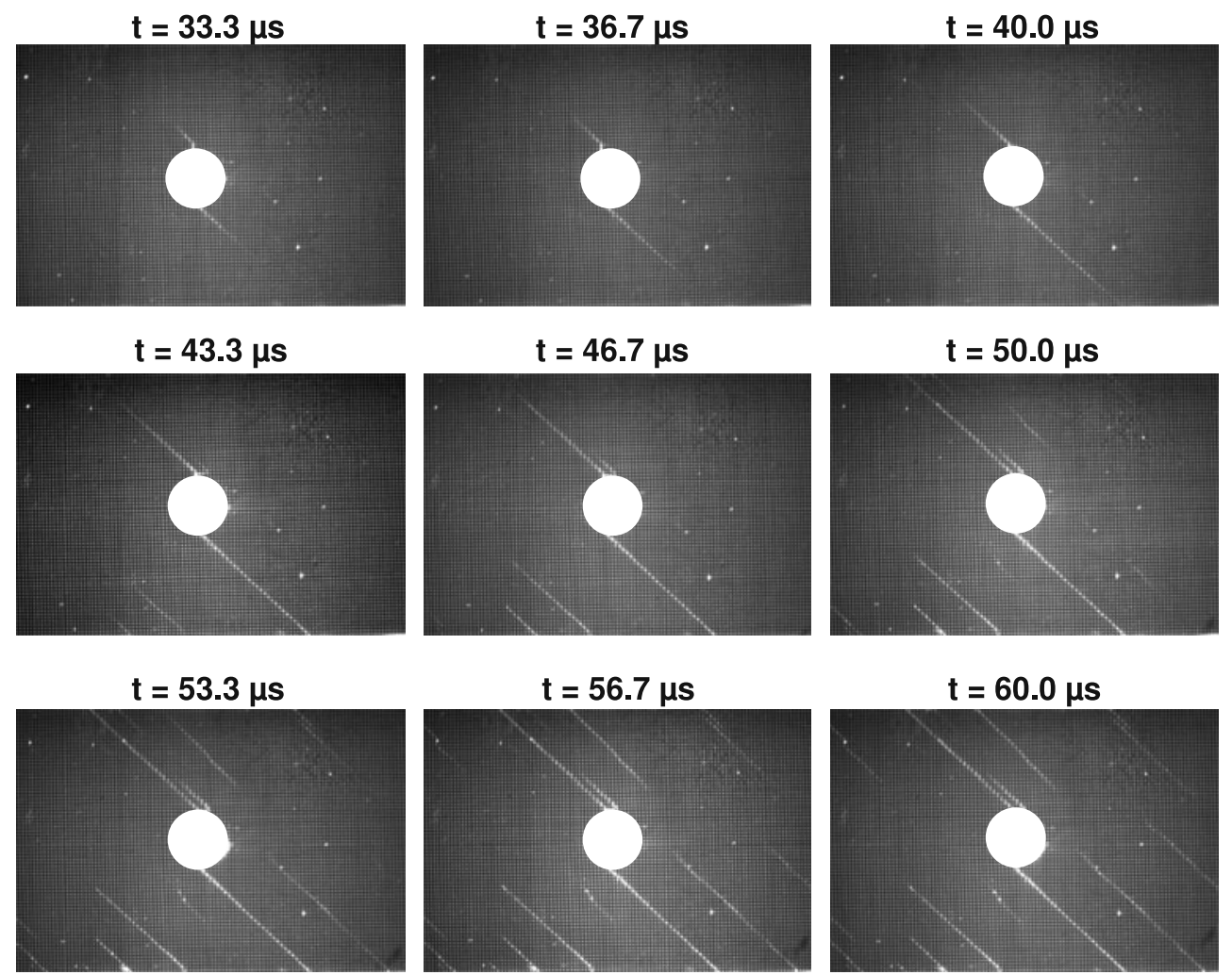
Fig. $10\left(U_{x}-\overline{U_{x}}\right)$ maps (colormap in $\mu \mathrm{m})$ (test 1 , tensile direction: horizontal, field of view: $20 \times 16 \mathrm{~mm}$ )
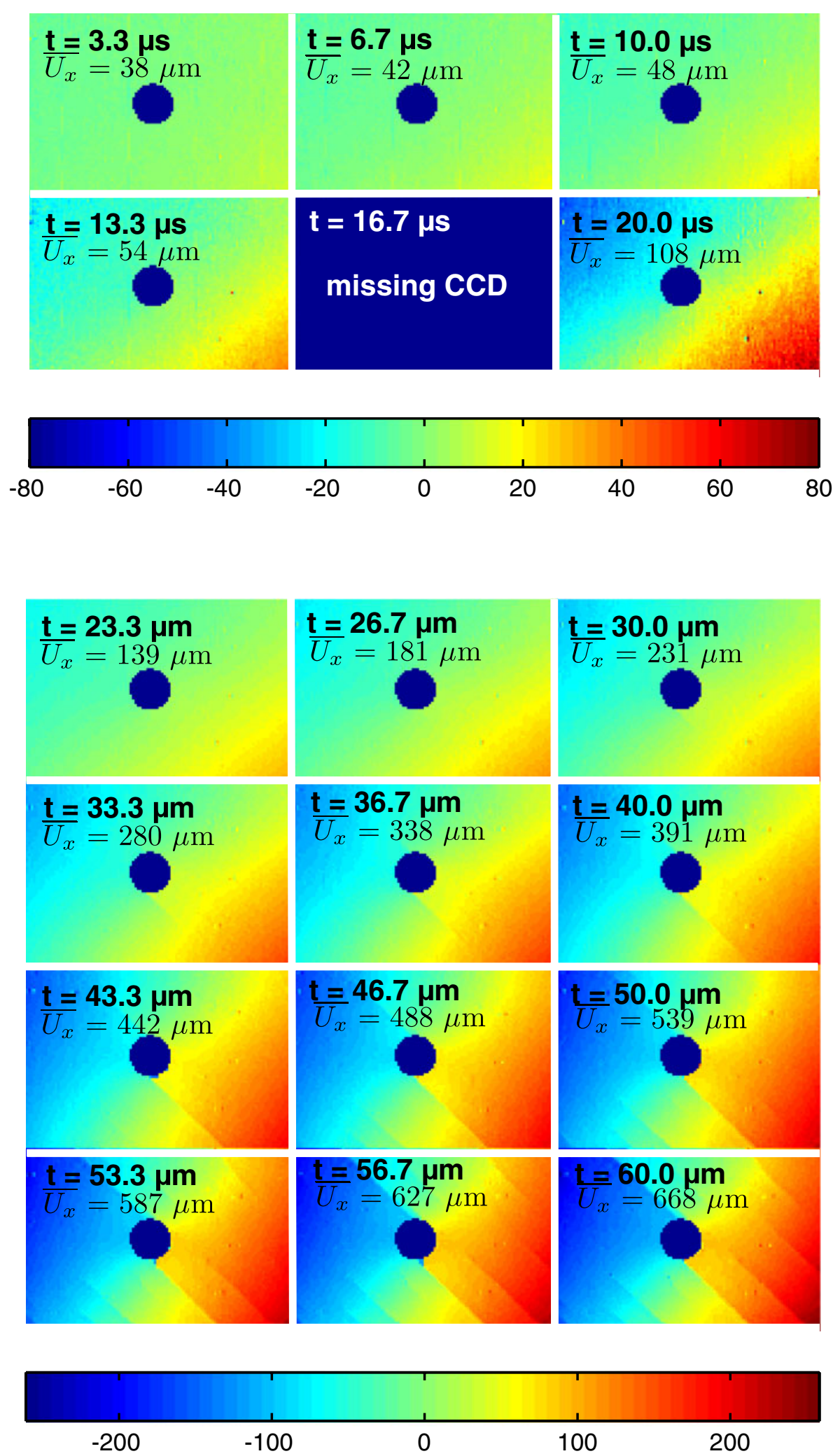
Fig. $11 \varepsilon_{x x}$ maps (test 1, tensile direction: horizontal, field of view: $20 \times 16 \mathrm{~mm}$ )
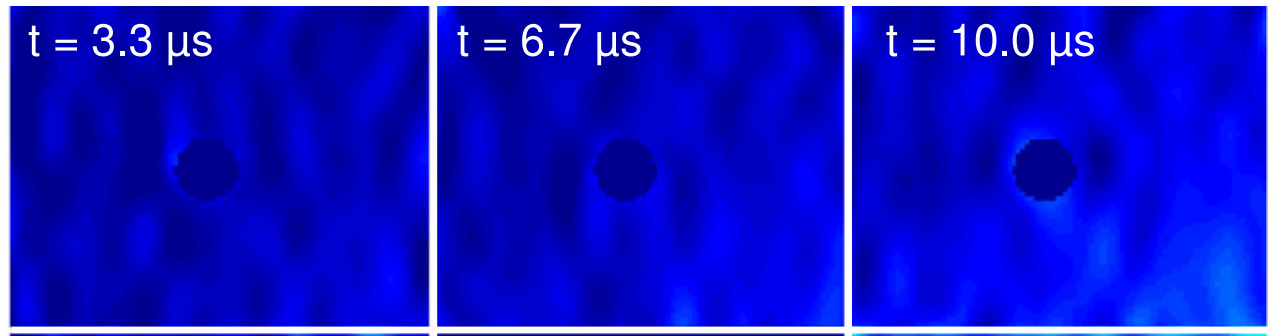

\section{$t=13.3 \mu s$}

$t=16.7 \mu s$

$\mathrm{t}=20.0 \mu \mathrm{s}$

$$
\begin{array}{l|l}
t=23.3 \mu s & t=26.7 \mu s
\end{array}
$$

\section{missing CCD}
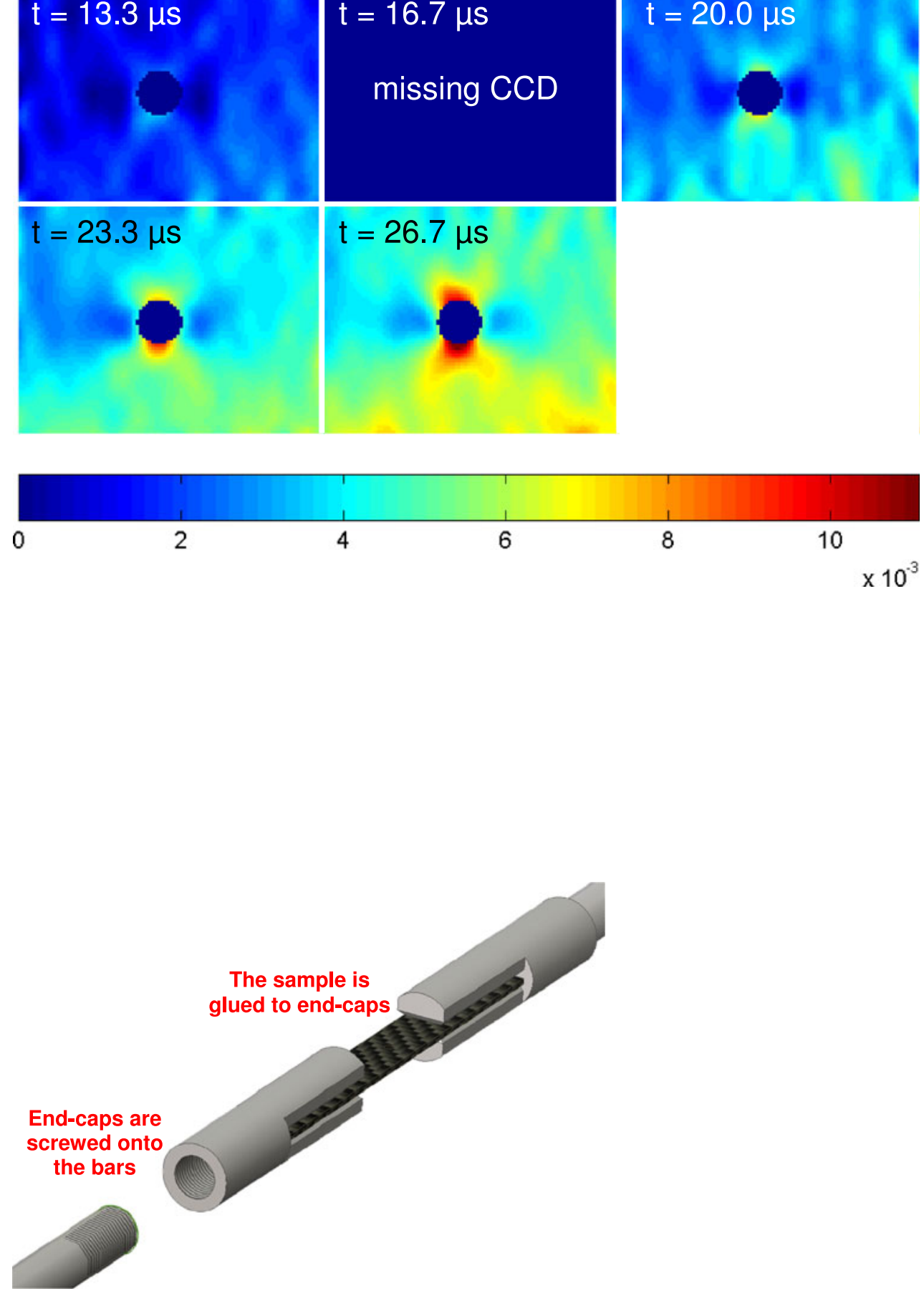

Fig. 12 Attachment of the sample to the SHPB set up 
Fig. $13 \dot{\varepsilon}_{x x}$ maps

(colormap in $\mathrm{s}^{-1}$ )

(test 1, tensile direction: horizontal, field of view: $20 \times 16 \mathrm{~mm}$ )
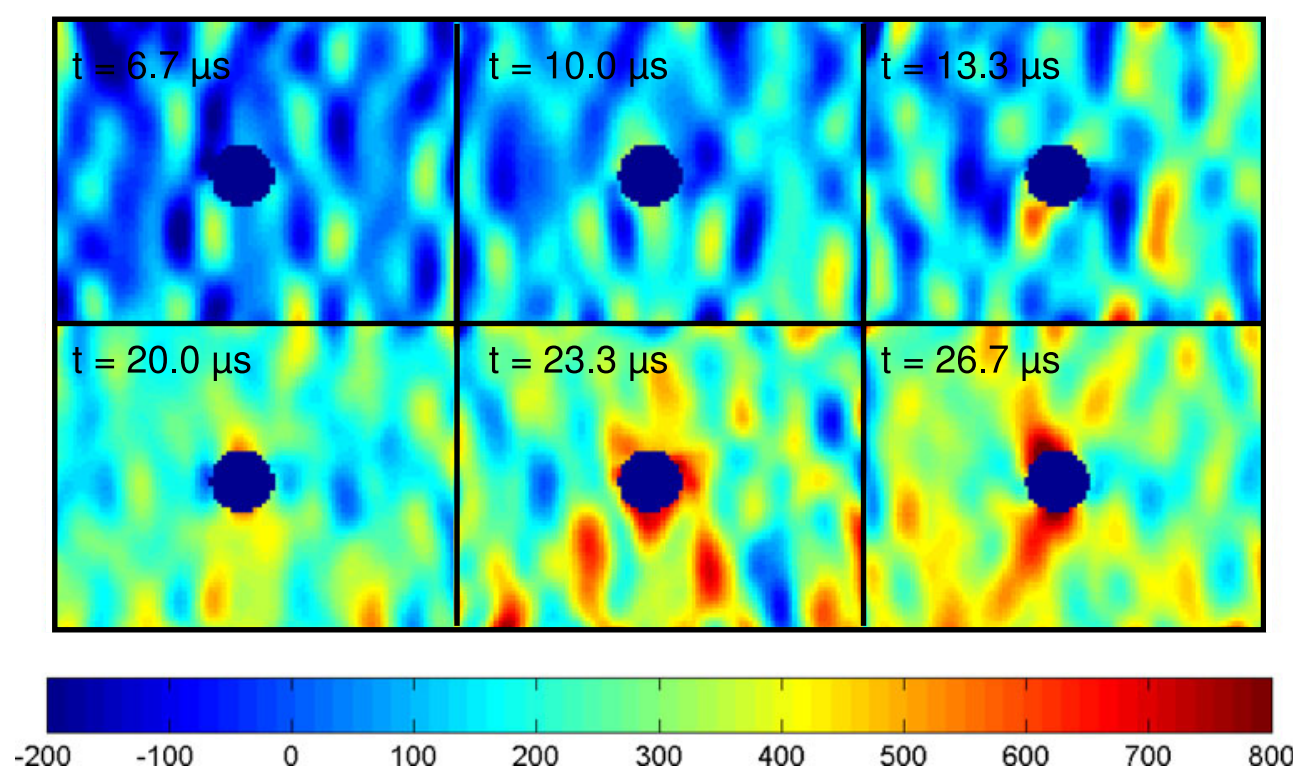

Second test: tensile coupon without hole

For this test too, a set of 62 frames has been recorded with the camera with a frequency of $300,000 \mathrm{fps}$. The time $t=0$ corresponds to frame 50. At the end of the recording $(t=46.3 \mu \mathrm{s})$, no crack at the surface of the sample has occurred.

The data given by two strain gauges glued onto the input and output bars and located at the same distance from the boundary of the sample (i.e., $730 \mathrm{~mm}$ ) have been recorded. This allowed to plot load curves using the following equation:

$F=E_{b} S_{b} \varepsilon$,

where $E_{b}$ is the Young's modulus of the material of the bar (titanium, $E_{b}=107 \mathrm{GPa}$ ), $S_{b}$ is the cross-section of the bar (here, circular with a diameter of $16 \mathrm{~mm}$ ) and $\varepsilon$ is
Fig. 14 Load curves obtained from strain gauges signal (test 2)

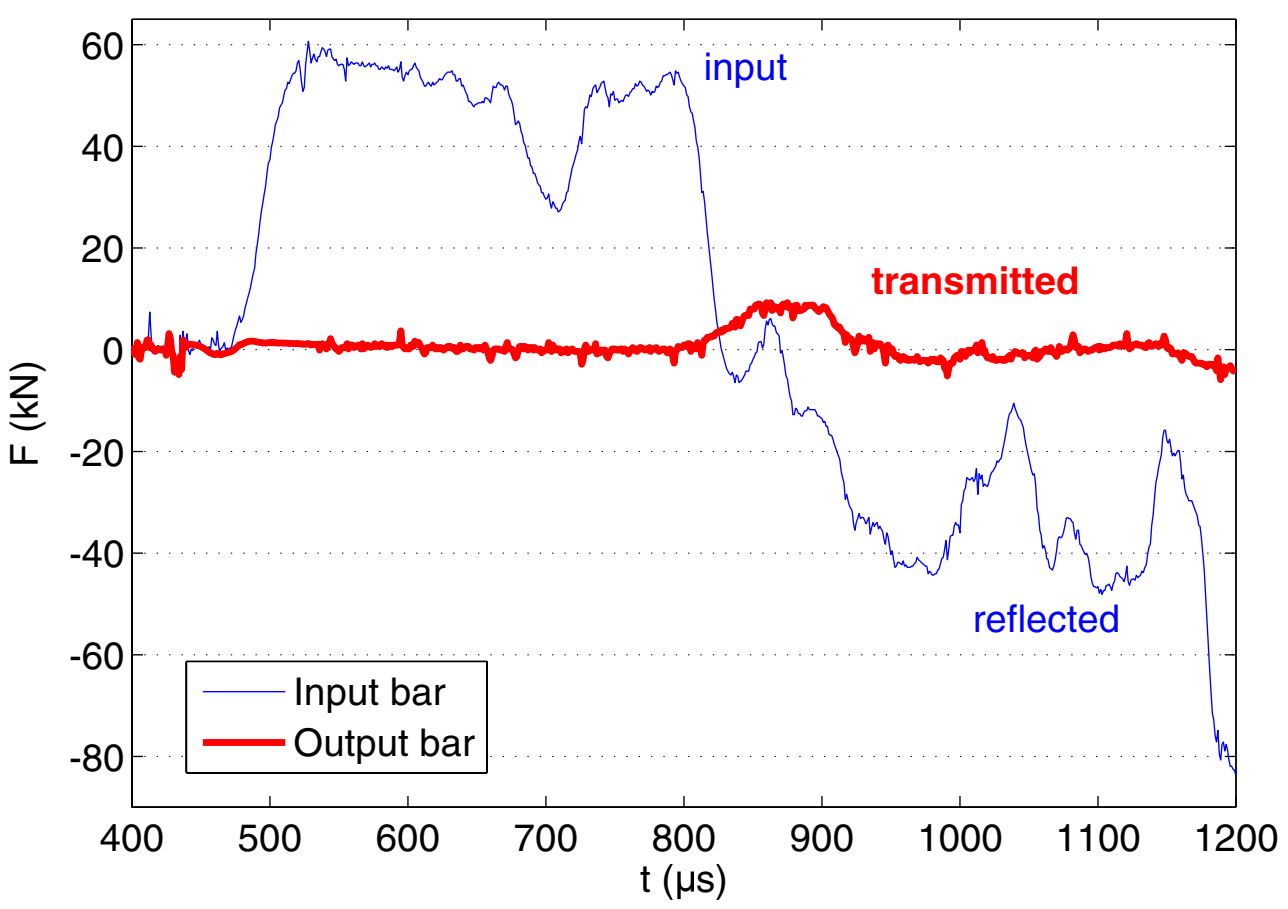


Fig. $15\left(U_{x}-\overline{U_{x}}\right)$ maps (colormap in $\mu \mathrm{m})$ (test 2, tensile direction: horizontal, field of view: $20 \times 16 \mathrm{~mm}$ )
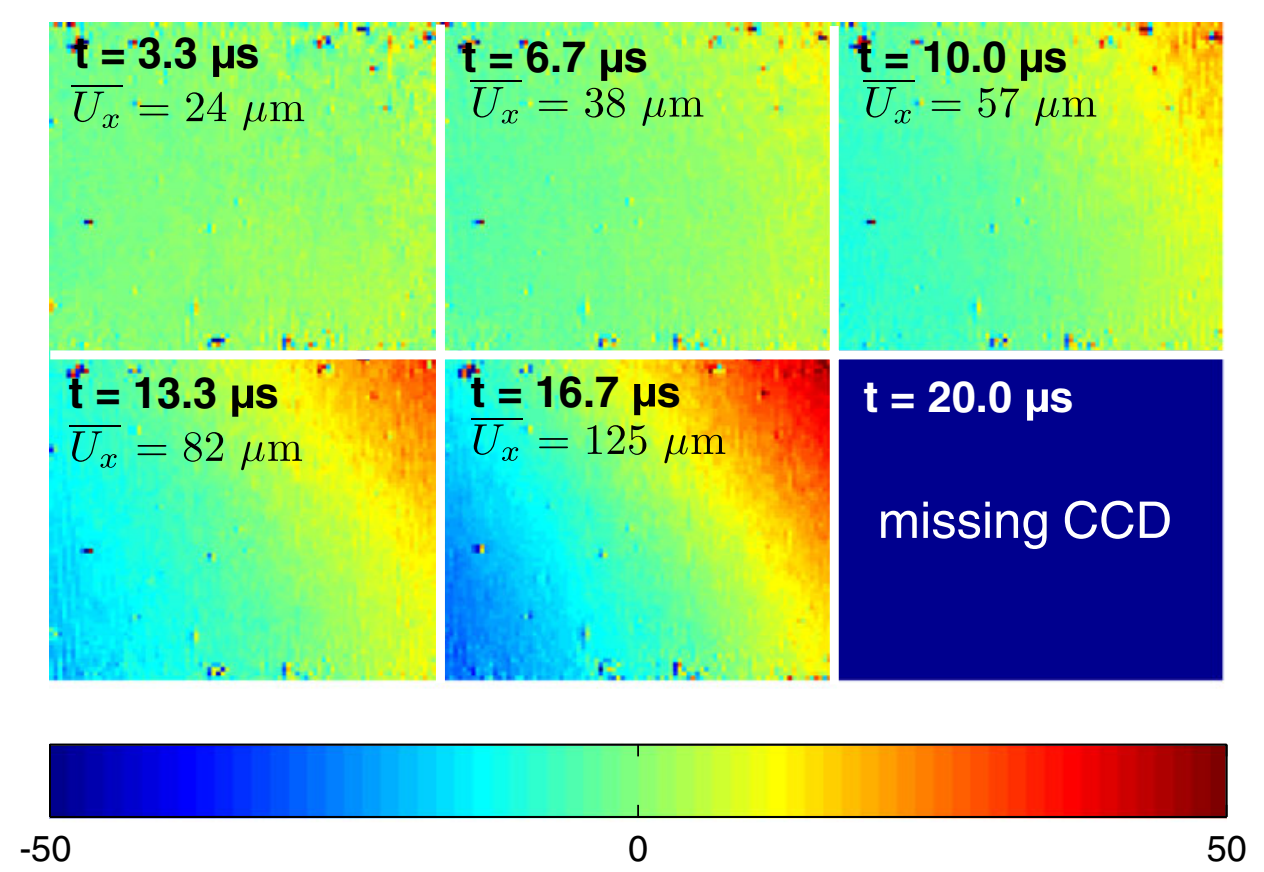

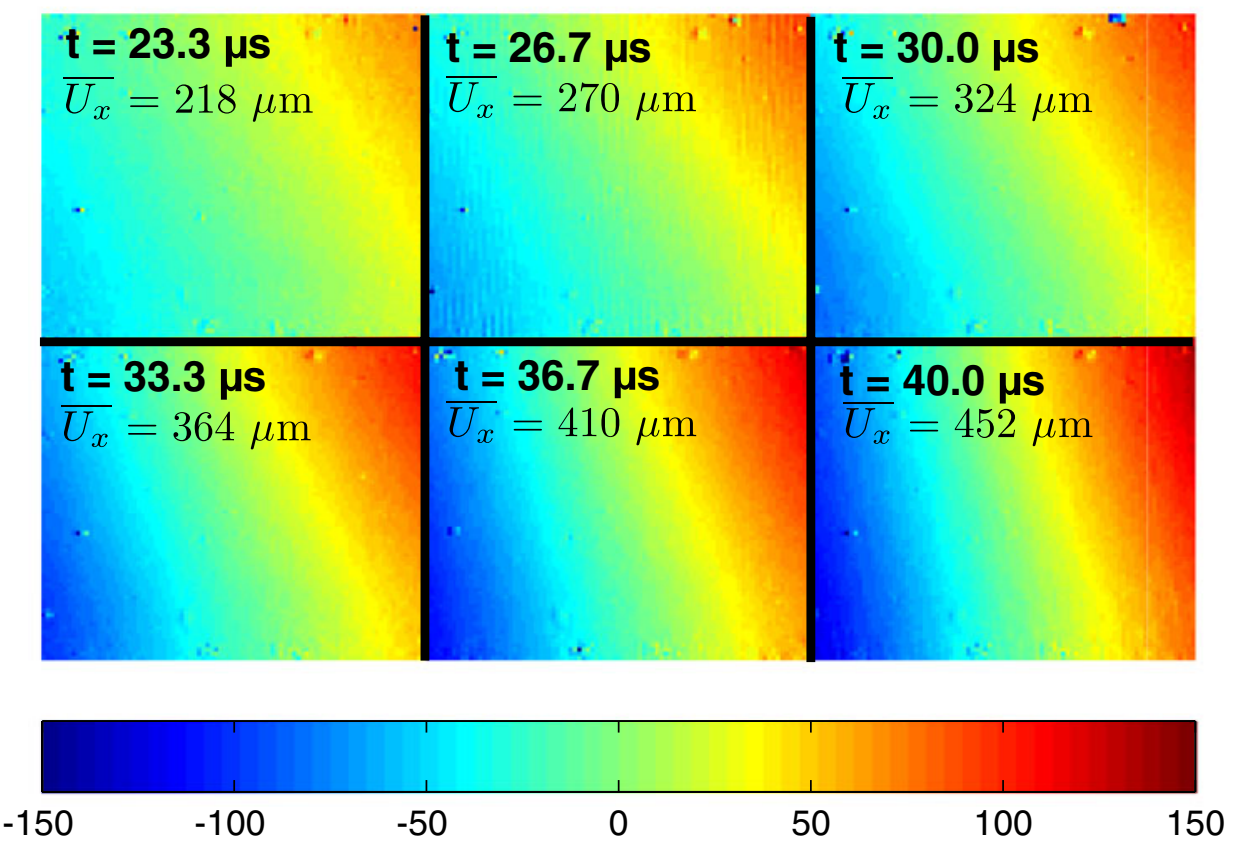

the recorded strain. The load curves are shown on Fig. 14 (on this figure, the time origin is not the same as the one mentioned previously). On the curve of the input load, many perturbations can be observed on the reflected wave. They are related to the interferences that occurred during the test in the screw joining the end-cap to the input bar. Indeed, between them, multiple rebounds created parasitic loads superimposed to the reflected wave leading to this disturbed shape of the curve.

Once again, the displacement maps show an inclination of the isodisplacement lines (Fig. 15). On the related strain maps (obtained by diffuse approximation, 
critical radius equal to 10), a slight bending effect can also be noted (Fig. 16). These observations seem to confirm that this phenomenon originates from the fixation of the samples to the bars.
From these two tests, it should be possible to identify the mechanical properties of the samples by using some inverse procedure. The next section is devoted to this identification.
Fig. $16 \varepsilon_{x x}$ maps (test 2, tensile direction: horizontal, field of view: $20 \times 16 \mathrm{~mm}$ )
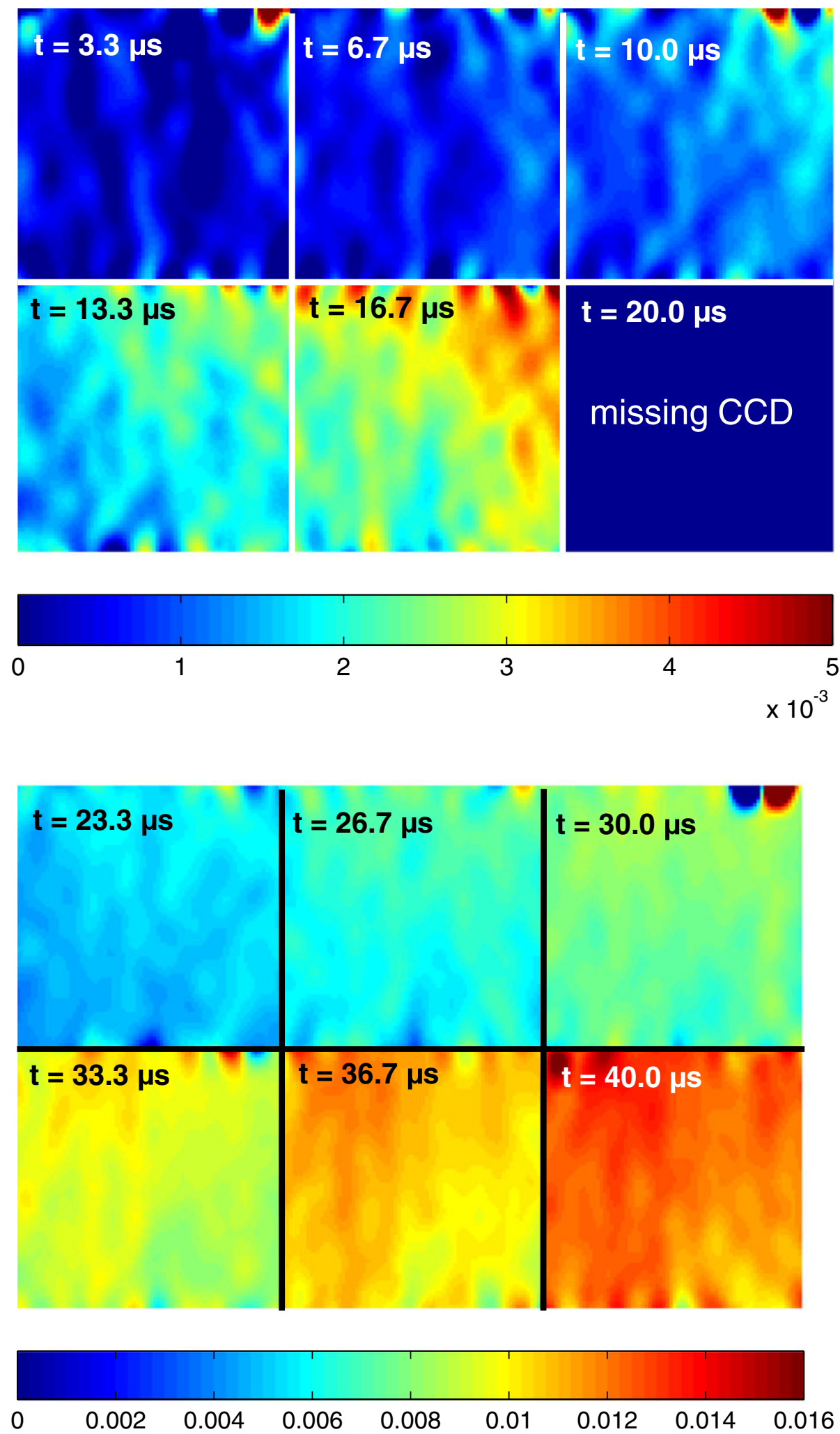


\section{Part II: Identification of Mechanical Properties Using the Virtual Fields Method}

Principle of the Use of the Virtual Fields Method at High Strain Rate to Identify Mechanical Properties Without Loading Information

As already mentioned, it is also possible to calculate acceleration maps from the displacements by double temporal differentiation. $a_{x}$ acceleration maps are shown on Figs. 17 and 18 respectively for the first test (between 6.7 and $26.7 \mu \mathrm{s}$ ) and for the second one (between 10.0 and $26.7 \mu \mathrm{s})$. They show a spatial localization following that of the displacement maps (Figs. 10 and 15) for the first stages of loading: the acceleration has propagated through the sample from the right to the left: it is not homogeneous but exhibits a gradient within the sample. These first stages are then followed by a second phase of acceleration decrease (that can be observed in the two last maps of Fig. 18) down to near zero where the sample is submitted to a constant velocity.

To check if these maps obtained for the interesting loading stages are significant or not, both the average reconstructed $U_{x}$ displacement and the average $a_{x}$ ac- celeration have been plotted for the first test. Figure 19 shows these curves and some maps corresponding to several loading stages: two before the occurrence of the deformation of the sample, two during the acceleration phase of the sample, and two after this phase. It appears clearly that the global level of acceleration is much higher between 6.7 and $26.7 \mu$ s and that the corresponding maps show a low frequency spatial signal of higher level than the others which allow to assert that, for these stages, the acceleration maps are indeed significant. The same kind of figure could be plotted for the second test.

Following these considerations on accelerations and strains, it should be possible to use them in order to identify the mechanical properties of the tested material. For this purpose, the Virtual Fields Method (VFM) has been used. This technique is based on the principle of virtual work $[33,39,40]$. In this dynamic case, this principle can be written as follows:
Fig. $17 a_{x}$ maps (colormap in $\mathrm{m} \cdot \mathrm{s}^{-2}$ ) (test 1 , tensile direction: horizontal, field of view: $20 \times 16 \mathrm{~mm}$ )
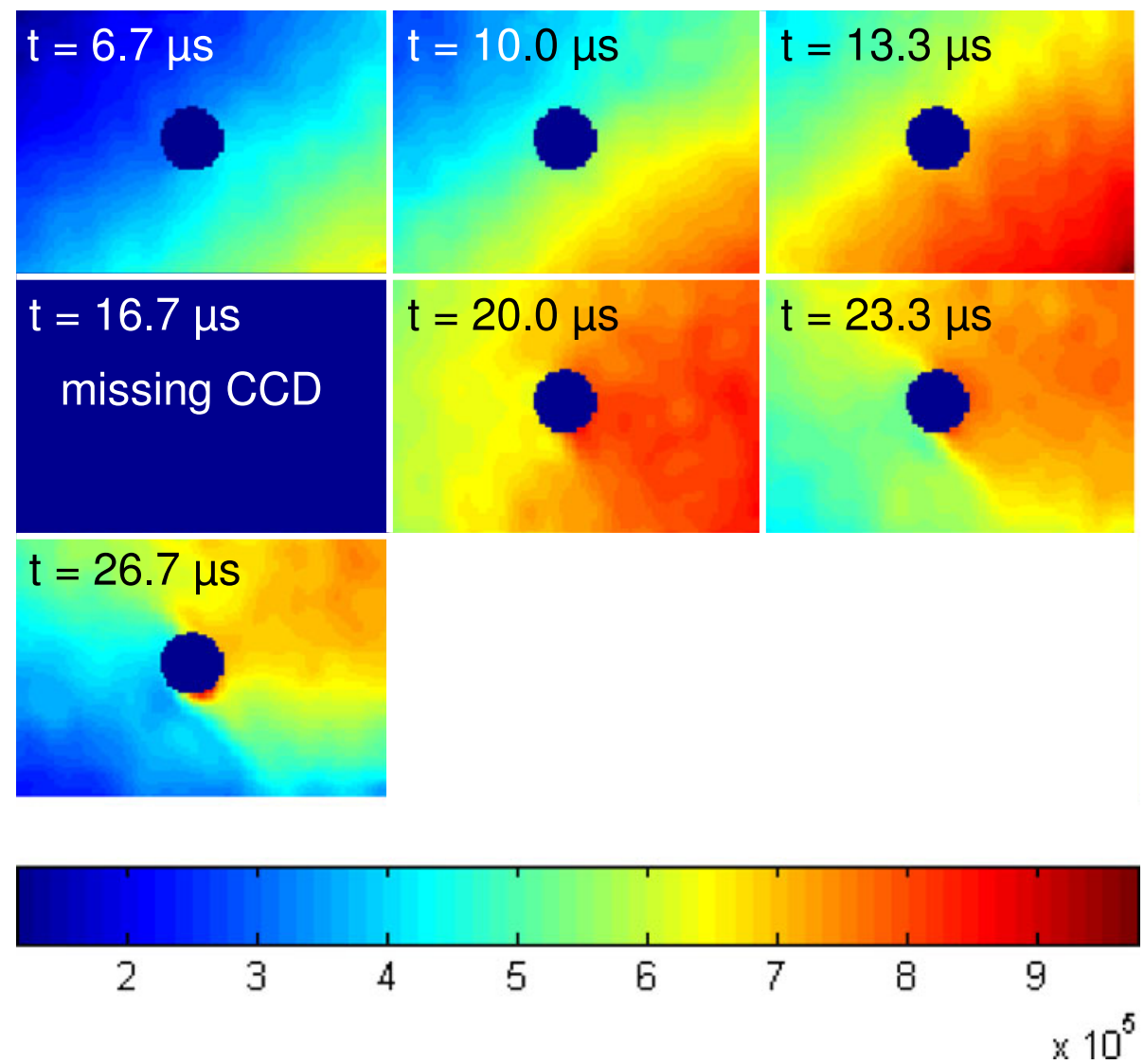
Fig. $18 a_{x}$ maps (colormap in $\mathrm{m} \cdot \mathrm{s}^{-2}$ ) (test 2 , tensile direction: horizontal, field of view: $20 \times 16 \mathrm{~mm}$ )
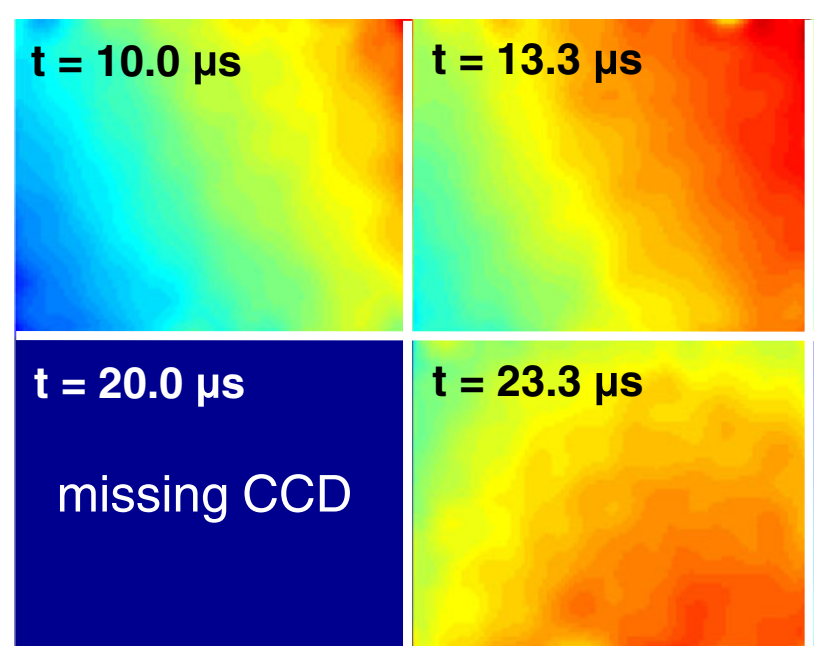

$t=16.7 \mu s$

$t=23.3 \mu s$

\section{$t=26.7 \mu s$}

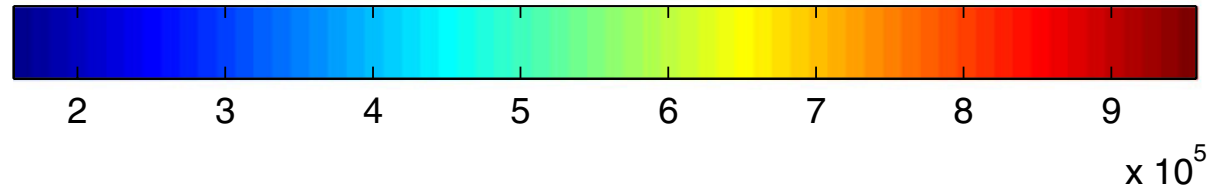

with:

- $\sigma$, the actual stress tensor;

- $\quad U^{*}$, a virtual displacement field kinematically admissible and $\varepsilon^{*}$, the virtual strain tensor deduced from it;

- $\quad V$, the volume of the region of interest;

- $T$ the actual loading imposed at the boundary of the considered region of interest $\partial V$;

- $\quad a$, the actual acceleration field;

- $\rho$, the density of the material;

- ":" is the contracted product between tensors and "." the dot product between vectors.

If $U^{*}$ is chosen such as it cancels the term $\int_{\partial V} T$. $U^{*} d S$ and if the acceleration $a$ is negligible (i.e., the term $\int_{V} \rho a \cdot U^{*} d V \simeq 0$ ), equation (4) becomes:

$\int_{V} \sigma: \varepsilon^{*} d V=0$

The stress field $\sigma$ can be related to the actual strain field $\varepsilon$ through the constitutive equation. In a planestress problem in linear orthotropic elasticity, this can be written as follows:

$\left(\begin{array}{c}\sigma_{x} \\ \sigma_{y} \\ \sigma_{s}\end{array}\right)=\left[\begin{array}{ccc}Q_{x x} & Q_{x y} & 0 \\ Q_{x y} & Q_{y y} & 0 \\ 0 & 0 & Q_{s s}\end{array}\right]\left(\begin{array}{l}\varepsilon_{x} \\ \varepsilon_{y} \\ \varepsilon_{s}\end{array}\right)$, with the subscript contraction convention $(x x \rightarrow x$, $y y \rightarrow y, x y \rightarrow s$ ). Equation (5) thus becomes:

$\sum_{i} \sum_{j} Q_{i j} \underbrace{\int_{V} \varepsilon_{j} \varepsilon_{i}^{*} d V}_{A_{i j}}=0, i=(x, y, s), j=(x, y, s)$.

Or, putting it into the form of a dot product of two vectors:

$\left\langle A_{i j}\right\rangle \cdot\left(Q_{i j}\right)=0, i=(x, y, s), j=(x, y, s)$.

By choosing as many virtual fields matching this condition $\int_{\partial V} T \cdot U^{*} d S=0$ as unknown rigidities ( 4 for linear elastic orthotropy) and always assuming that $a \simeq$ 0 , it leads to a system of 4 linear equations with 4 unknowns that can be written:

$[A](Q)=(0)$.

This approach would lead to relative values of the stiffnesses $Q$ only (which is logical as no loading is involved to determine their order of magnitude). In the particular case of an isotropic behaviour, equation (9) would give access to the knowledge of $v$, Poisson's ratio, but not to Young's modulus.

In the general case, to determine the absolute values of $Q$, the knowledge of the loading is needed. In a quasi-static loading case (i.e., $a \simeq 0$ ), equation (4) can be written:

$\int_{V} \sigma: \varepsilon^{*} d V=\int_{\partial V} T \cdot U^{*} d S$ 
Fig. 19 Average

reconstructed $U_{x}$ and average $a_{x}$ plotted against time and six $a_{x}$ acceleration maps (colormap in $\mathrm{m} \cdot \mathrm{s}^{-2}$ ) (test 1$)$

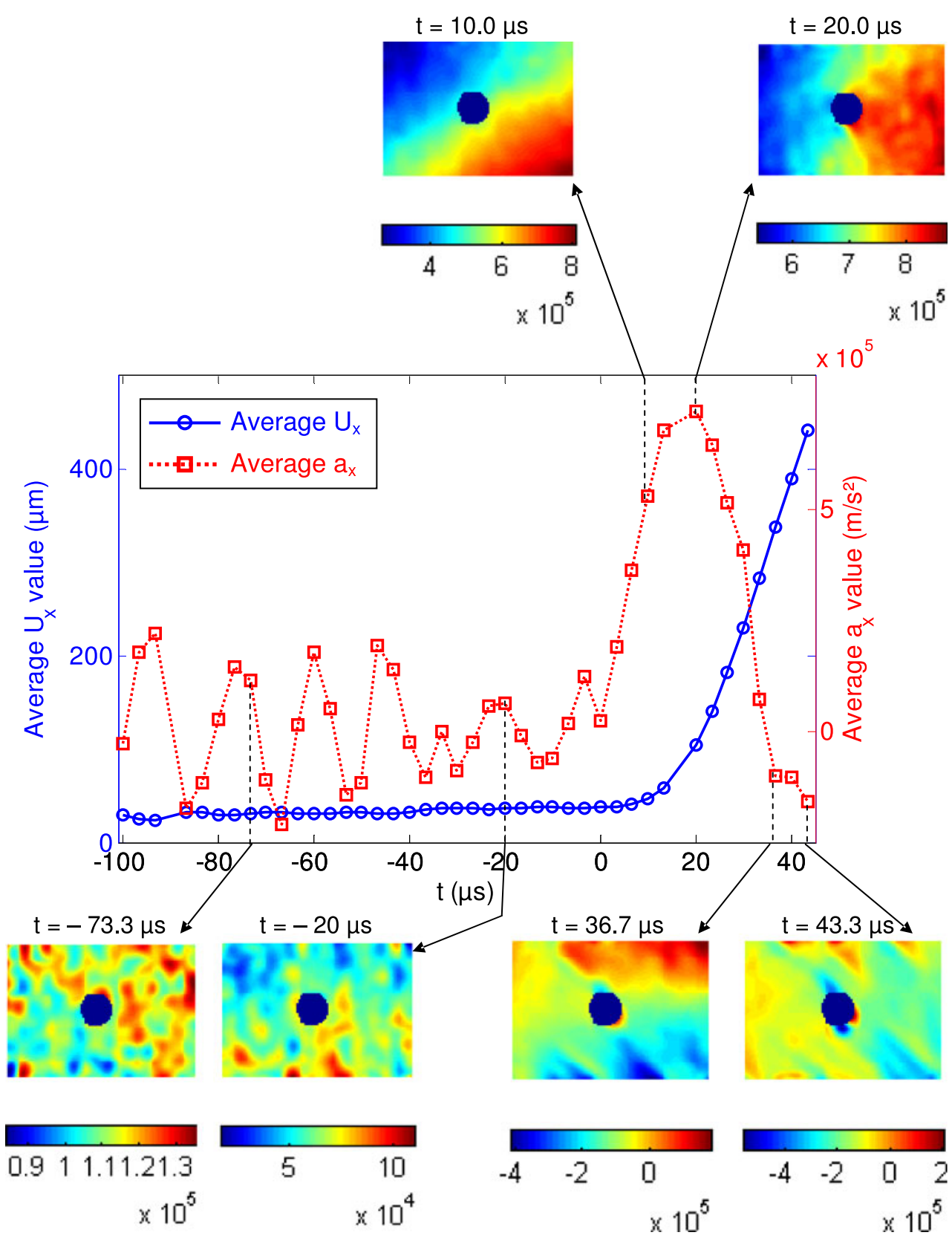

In a dynamic case, if the actual acceleration is significant enough, the knowledge of the loading is no longer needed: by choosing virtual fields $U^{*}$ such that $\int_{\partial V} T \cdot U^{*} d S=0$, equation (4) becomes:

$-\int_{V} \sigma: \varepsilon^{*} d V=\int_{V} \rho a \cdot U^{*} d V$

In this case, the inertial forces act as a distributed load cell (assuming that the density $\rho$ is known and constant all over the duration of the mechanical test). This approach has already been successfully applied in vibration to identify damping properties of thin plates [41-45] but never to a high strain rate loading cases.

Identification of the Quasi-Isotropic Stiffness Components of the Sample

In the present study, the composite sample has a quasiisotropic membrane behaviour. The constitutive equation (6) thus becomes:

$$
\left(\begin{array}{c}
\sigma_{x} \\
\sigma_{y} \\
\sigma_{s}
\end{array}\right)=\left[\begin{array}{ccc}
Q_{x x} & Q_{x y} & 0 \\
Q_{x y} & Q_{x x} & 0 \\
0 & 0 & \frac{Q_{x x}-Q_{x y}}{2}
\end{array}\right]\left(\begin{array}{c}
\varepsilon_{x} \\
\varepsilon_{y} \\
\varepsilon_{s}
\end{array}\right),
$$


with:

$Q_{x x}=\frac{E}{1-v^{2}} ; Q_{x y}=\frac{v E}{1-v^{2}}=v Q_{x x}$,

where $E$ is Young's modulus and $v$, Poisson's ratio.

The two unknown stiffnesses $Q_{x x}$ and $Q_{x y}$ have been identified using the inertial forces as explained above. To reach this purpose, two independent virtual fields, $\left\{\left(U_{x_{i}}^{*}, U_{y_{i}}^{*}\right) ;\left(\varepsilon_{x_{i}}^{*}, \varepsilon_{y_{i}}^{*}, \varepsilon_{s_{i}}^{*}\right)\right\}, i \in\{1,2\}$ had to be chosen such as the condition $\int_{\partial V} T \cdot U^{*} d S=0$ is fulfilled. Indeed, with the SHPB set up, the resultant of the loading is usually measured. Thus, virtual fields resulting in a non zero virtual work of these external forces could be selected i.e., a $U^{*}$ field constant on the edges of the sample such as:

$$
\int_{\partial V} T \cdot U^{*} d S=U^{*} \cdot \underbrace{\int_{\partial V} T d S .}_{\text {resultant }}
$$

However, load is very difficult to measure in dynamics because of inertial effects (so-called "non equilibrium" phase which should really be called "non quasi-static equilibrium" phase). Moreover, the classical SHPB analysis requires a rather cumbersome set up and very restrictive assumptions in terms of specimen geometry. The idea of this paper is to explore the use of acceleration forces as an alternative. This is the reason why here, the term $\int_{\partial V} T \cdot U^{*} d S$ has been canceled out. To reach this purpose, $U^{*}$ has to be null on the edges as shown on Fig. 20. An example of two fields that match this condition is: ${ }^{1}$

Field $\mathrm{n}^{\circ} 1\left\{\begin{array}{l}U_{x_{1}}^{*}=x(x-L) \\ U_{y_{1}}^{*}=0\end{array} \Rightarrow\left\{\begin{array}{l}\varepsilon_{x_{1}}^{*}=2 x-L \\ \varepsilon_{y_{1}}^{*}=0 \\ \varepsilon_{s_{1}}^{*}=0\end{array}\right.\right.$

Field $n^{\circ} 2\left\{\begin{array}{l}U_{x_{2}}^{*}=0 \\ U_{y_{2}}^{*}=x(x-L) y\end{array} \Rightarrow\left\{\begin{array}{l}\varepsilon_{x_{2}}^{*}=0 \\ \varepsilon_{y_{2}}^{*}=x(x-L) \\ \varepsilon_{s_{2}}^{*}=(2 x-L) y\end{array}\right.\right.$

\footnotetext{
${ }^{1}$ It has to be underlined that, normally, the virtual fields are chosen automatically to ensure their maximum independence as explained in [46, 47]. But since this automatic procedure has not been implemented for dynamic cases yet, the two fields are chosen manually here. This is all the more reasonable since only two unknowns have to be identified.
}

These two fields correspond to the best choice of virtual fields here as they present the lowest degree of polynomial compatible with the conditions previously mentioned. Indeed, previous experience with the VFM has shown that, generally, the simplest the virtual fields, the more relevant the identification results.

With these two fields, a linear system of two equations with two unknowns can be obtained:

$\left[\begin{array}{ll}A_{11} & A_{12} \\ A_{21} & A_{22}\end{array}\right]\left(\begin{array}{l}Q_{x x} \\ Q_{x y}\end{array}\right)=\left(\begin{array}{l}B_{1} \\ B_{2}\end{array}\right)$

with:

$\left\{\begin{array}{l}A_{i 1}=\int_{V}-\left(\varepsilon_{x} \varepsilon_{x_{i}}^{*}+\varepsilon_{y} \varepsilon_{y_{i}}^{*}+\frac{1}{2} \varepsilon_{s} \varepsilon_{s_{i}}^{*}\right) d V ; \\ A_{i 2}=\int_{V}-\left(\varepsilon_{y} \varepsilon_{x_{i}}^{*}+\varepsilon_{x} \varepsilon_{y_{i}}^{*}-\frac{1}{2} \varepsilon_{s} \varepsilon_{s_{i}}^{*}\right) d V ; \\ B_{i}=\int_{V} \rho\left(a_{x} U_{x_{i}}^{*}+a_{y} U_{y_{i}}^{*}\right) d V .\end{array}\right.$

These integrals are calculated assuming that the strains are constant within the thickness. In this case, the first term of equation (17) for instance becomes:

$A_{i 1}=t \int_{S}-\left(\varepsilon_{x} \varepsilon_{x_{i}}^{*}+\varepsilon_{y} \varepsilon_{y_{i}}^{*}+\frac{1}{2} \varepsilon_{S} \varepsilon_{S_{i}}^{*}\right) d S$

where $t$ represents the thickness and $S$, the area of the region of interest where strain fields are measured. Practically, these continuous integrals are calculated as discrete sums as:

$$
\begin{aligned}
A_{i 1} & =t \int_{S}-\left(\varepsilon_{x} \varepsilon_{x_{i}}^{*}+\varepsilon_{y} \varepsilon_{y_{i}}^{*}+\frac{1}{2} \varepsilon_{S} \varepsilon_{s_{i}}^{*}\right) d S \\
& =t \sum_{\text {ROI }}-\left(\varepsilon_{x} \varepsilon_{x_{i}}^{*}+\varepsilon_{y} \varepsilon_{y_{i}}^{*}+\frac{1}{2} \varepsilon_{S} \varepsilon_{s_{i}}^{*}\right) \times p^{2} .
\end{aligned}
$$

"ROI" denotes the whole number of measurement points on the region of interest and $p$ is the size of one measurement point such as $p^{2}$ is its area (here $p$ is equal to the period of the grid).

The linear system of equation (16) can then be inverted (assuming that the $A$ matrix is invertible which depends on the accurate choice of the virtual fields) 
Fig. 20 Conditions to choose the virtual fields (in order to cancel the virtual work of the applied loading)

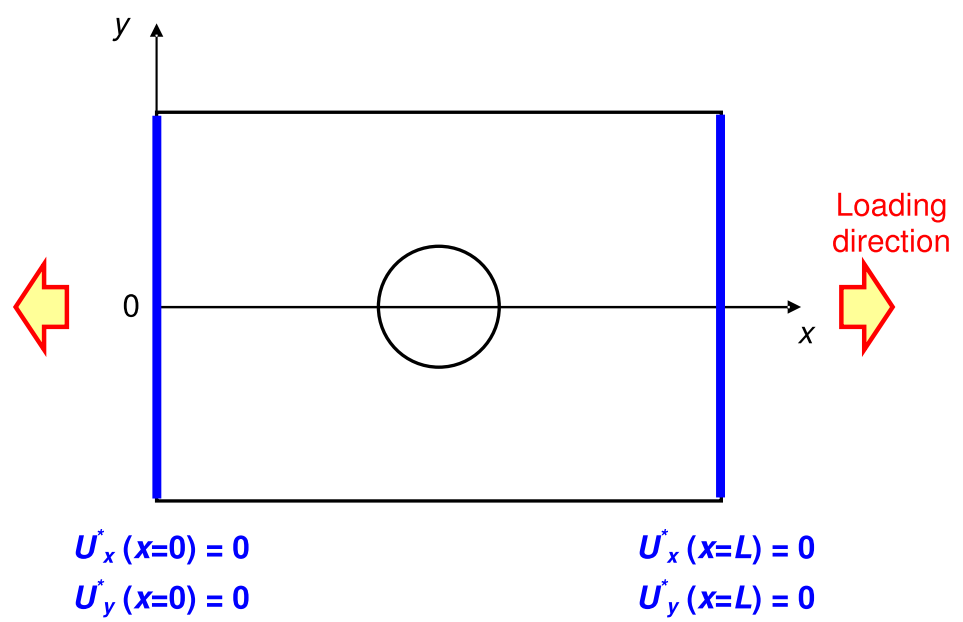

Virtual field 1: $\mathrm{U}_{\mathrm{x}}^{*}=\mathrm{x}(\mathrm{x}-\mathrm{L}) ; \mathrm{U}_{\mathrm{y}}^{*}=0$

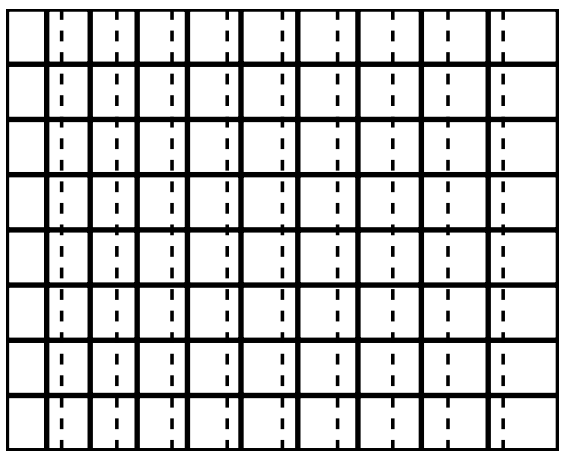

and the unknown stiffnesses are directly given by the following equation:

$$
\left(\begin{array}{l}
Q_{x x} \\
Q_{x y}
\end{array}\right)=\left[\begin{array}{ll}
A_{11} & A_{12} \\
A_{21} & A_{22}
\end{array}\right]^{-1}\left(\begin{array}{l}
B_{1} \\
B_{2}
\end{array}\right)
$$

It should be noted that, in the present study, the acceleration term in the $y$ direction is negligible compared to that in the $x$ direction $\left(a_{y} \simeq 10^{4} \mathrm{~m} \cdot \mathrm{s}^{-2}\right.$ and $a_{x} \simeq 10^{6}$ $\mathrm{m} \cdot \mathrm{s}^{-2}$ ). Therefore, the term $B_{2}$ in equation (20) can be ignored here (this was checked with the present data) and this equation can be written:

$$
\left(\begin{array}{l}
Q_{x x} \\
Q_{x y}
\end{array}\right)=\left[\begin{array}{ll}
A_{11} & A_{12} \\
A_{21} & A_{22}
\end{array}\right]^{-1}\left(\begin{array}{c}
B_{1} \\
0
\end{array}\right)
$$

In these conditions, the VFM leads to a set of two uncoupled equations: the second one gives access to Poisson's ratio and then, the first one one, to Young's modulus. Especially, the acceleration maps do not have any influence on the identification of Poisson's ratio $\left(v=-A_{21} / A_{22}\right)$.

This method should work very well whenever significant acceleration and strain signals are available that is to say in the present case for times between 6.7 and $26.7 \mu$ s for the first test and between 10.0 and $26.7 \mu$ s for the second one. The identified properties obtained for these stages with the virtual fields mentioned in equation (15) are given in Tables 1 and 2. Although these values are not exactly the expected ones (reference values given in Tables 1 and 2), ${ }^{2}$ it appears that the proposed approach gives access to the right

\footnotetext{
${ }^{2}$ These values are calculated from classical lamination theory from the stacking sequence and the following ply properties: $E_{11}=43.9 \mathrm{GPa}, E_{22}=15.4 \mathrm{GPa}, v_{12}=0.3, G_{12}=4.34 \mathrm{GPa}$, obtained from usual quasi-static standard tests on specimens made from the same prepreg [48]. There are unfortunately no directly measured reference values available for this material at the strain rates tested here.
} 
Table 1 Identified mechanical properties-test 1

\begin{tabular}{|c|c|c|c|c|c|c|}
\hline \multicolumn{6}{|c|}{ Time $(\mu s)$} & \multirow[t]{2}{*}{ Average } \\
\hline 6.7 & 10.0 & 13.3 & 20.0 & 23.3 & 26.7 & \\
\hline \multicolumn{7}{|c|}{ Young's modulus in GPa (reference value: $E=23.2 \mathrm{GPa}$ ) } \\
\hline 20.4 & 21.1 & 15.8 & 15.7 & 16.6 & 13.6 & 17.2 \\
\hline \multicolumn{7}{|c|}{ Poisson's ratio (reference value: $v=0.34$ ) } \\
\hline 0.28 & 0.22 & 0.32 & 0.27 & 0.29 & 0.30 & 0.28 \\
\hline
\end{tabular}

order of magnitude. ${ }^{3}$ It can be seen that the identified values of Young's modulus are generally lower than the reference ones. It is unlikely that this reduction is due to the strain rate effect, indeed, most studies mention an increase of the stiffnesses with the strain rate for such materials. So one could partially conclude that this gap between the values comes from the quality of the measurements. In particular, the acceleration data used to derive Young's modulus is very sensitive to noise (as it is obtained by double temporal differentiation). Indeed, it can be observed that the values of Poisson's ratio, that do not require the knowledge of the acceleration to be identified as explained previously, are not so far from the expected ones. For a better understanding of this phenomenon, the stability of the identified results is studied in the next section.

It should also be noted that the strain rate is not homogeneous within the specimen (especially for the first test due to the presence of the hole, see Fig. 13). Taking advantage of this heterogeneity, a strain rate sensitivity of the mechanical properties could be identified using the VFM as it is done in [6]. But, unfortunately here, the strain-rate resolution is not good enough to do it. Consequently, the study of this influence is one perspective of this work.

\section{Stability of the Identified Mechanical Parameters}

In order to address the stability of the results in a pragmatic way, several choices of different parameters have been tried out and reported in this section. If the results are stable, it allows to think that they are not only dependent on the choice of a "good" set of parameters and it gives confidence to their relevancy.

\footnotetext{
${ }^{3}$ It has to be underlined that the last identified Young's modulus for the second test (at time $t=26.7 \mu \mathrm{m}$ ) is rather far from the reference value. This can be explained by the fact that, at this time, the global acceleration is already low.
}

Table 2 Identified mechanical properties-test 2

\begin{tabular}{|c|c|c|c|c|c|}
\hline \multicolumn{5}{|c|}{ Time $(\mu \mathrm{s})$} & \multirow[t]{2}{*}{ Average } \\
\hline 10.0 & 13.3 & 16.7 & 23.3 & 26.7 & \\
\hline \multicolumn{6}{|c|}{ Young's modulus in GPa (reference value: $E=23.2 \mathrm{GPa}$ ) } \\
\hline 22.6 & 31.8 & 23.8 & 22.0 & 42.2 & 28.5 \\
\hline \multicolumn{6}{|c|}{ Poisson's ratio (reference value: $v=0.34$ ) } \\
\hline 0.46 & 0.31 & 0.34 & 0.3 & 0.32 & 0.35 \\
\hline
\end{tabular}

\section{Effect of the choice of the virtual fields}

The choice of the virtual fields can influence the relevancy of the results. To check this effect, other sets of virtual fields can be chosen, for instance:

Field $n^{\circ} 1\left\{\begin{array}{l}U_{x_{1}}^{*}=x(x-L)^{2} \\ U_{y_{1}}^{*}=0\end{array} \Rightarrow\left\{\begin{array}{l}\varepsilon_{x_{1}}^{*}=(x-L)(3 x-L) \\ \varepsilon_{y_{1}}^{*}=0 \\ \varepsilon_{s_{1}}^{*}=0\end{array}\right.\right.$

Field $\mathrm{n}^{\circ} 2\left\{\begin{array}{l}U_{x_{2}}^{*}=0 \\ U_{y_{2}}^{*}=x(x-L) y\end{array} \Rightarrow\left\{\begin{array}{l}\varepsilon_{x_{2}}^{*}=0 \\ \varepsilon_{y_{2}}^{*}=x(x-L) \\ \varepsilon_{s_{2}}^{*}=(2 x-L) y\end{array}\right.\right.$

Field $n^{\circ} 1\left\{\begin{array}{l}U_{x_{1}}^{*}=\sin \left(\frac{\pi x}{L}\right) \\ U_{y_{1}}^{*}=0\end{array} \Rightarrow\left\{\begin{array}{l}\varepsilon_{x_{1}}^{*}=\frac{\pi}{L} \times \cos \left(\frac{\pi x}{L}\right) \\ \varepsilon_{y_{1}}^{*}=0 \\ \varepsilon_{s_{1}}^{*}=0\end{array}\right.\right.$

Field $\mathrm{n}^{\circ} 2\left\{\begin{array}{l}U_{x_{2}}^{*}=0 \\ U_{y_{2}}^{*}=x(x-L) y\end{array} \Rightarrow\left\{\begin{array}{l}\varepsilon_{x_{2}}^{*}=0 \\ \varepsilon_{y_{2}}^{*}=x(x-L) \\ \varepsilon_{s_{2}}^{*}=(2 x-L) y\end{array}\right.\right.$

On these two sets of virtual fields expressed in equations (22) and (23) (respectively referred as "VF set 2" and "VF set 3"), only the first one has been changed compared to the virtual fields expressed in equation (15) as this one leads to the identification of $E$. Two new sets of parameters can thus be identified for the first test for instance (Table 3).

It can be seen that the results obtained for VF set 1 and VF set 3 are very close to each other whereas the results obtained for VF set 2 are significantly different (for Young's modulus values). It can be explained by the fact that the expression of $U_{x_{1}}^{*}$ for VF set 2 presents a higher spatial frequency signal than the two others that can lead to an instability of the identified results. This was already observed in the past. Nevertheless, the fact that the two other sets of results are strongly in accordance allows to think that the identification procedure is reliable. 
Table 3 Identified mechanical properties-test 1 -obtained with different virtual fields sets

\begin{tabular}{|c|c|c|c|c|c|c|c|}
\hline & \multicolumn{6}{|c|}{ Time $(\mu \mathrm{s})$} & \multirow[t]{2}{*}{ Average } \\
\hline & 6.7 & 10.0 & 13.3 & 20.0 & 23.3 & 26.7 & \\
\hline \multicolumn{8}{|c|}{ Young's modulus in $\mathrm{GPa}$ (reference value: $E=23.2 \mathrm{GPa}$ ) } \\
\hline VF Set 1 & 20.4 & 21.1 & 15.8 & 15.7 & 16.6 & 13.6 & 17.2 \\
\hline VF Set 2 & 33.8 & 27.9 & 15.5 & 18.2 & 23.3 & 12.5 & 21.8 \\
\hline VF Set 3 & 19.7 & 21.1 & 15.9 & 15.7 & 16.6 & 14.1 & 17.2 \\
\hline \multicolumn{8}{|c|}{ Poisson's ratio (reference value: $v=0.34$ ) } \\
\hline VF Set 1 & 0.28 & 0.22 & 0.32 & 0.27 & 0.29 & 0.30 & 0.28 \\
\hline VF Set 2 & 0.28 & 0.22 & 0.32 & 0.27 & 0.29 & 0.30 & 0.28 \\
\hline VF Set 3 & 0.28 & 0.22 & 0.32 & 0.27 & 0.29 & 0.30 & 0.28 \\
\hline
\end{tabular}

\section{Effect of noisy data}

To check the stability of the results against the quality of the measurements, experimental maps can be disturbed with additional simulated noise. Especially, as mentioned in "Noise study-experimental procedure", the acceleration maps present a bias that can reach up to $4 \cdot 10^{5} \mathrm{~m} \cdot \mathrm{s}^{-2}$. To study the influence of this potential bias on the identified values, two systematic errors of $1 \cdot 10^{5}$ and $2 \cdot 10^{5} \mathrm{~m} \cdot \mathrm{s}^{-2}$ have been added to $a_{x}$ maps. Table 4 shows the corresponding results (for the first test, obtained with VF set 1). They confirm that the identified $E$ value directly depends on the accuracy of the determination of the acceleration maps. Thus, it can be supposed that the gap between the identified Young's modulus for "raw acceleration maps" and its reference value is due to the fact that the acceleration is underestimated by the chosen algorithm (because of the polynomial smoothing).

\section{Effect of the spatial smoothing}

In order to study the influence of the spatial smoothing induced by the diffuse approximation algorithm, several critical radii have been used to smooth the measurements of the first test (as the spatial resolution is not a crucial problem for the second test since the loading state is more or less homogeneous as the sample does not present any hole).
The identification results are given in Table 5. They show that the spatial smoothing does not globally affect the identification procedure. By looking more precisely, the larger the radius, the higher Young's modulus. Indeed, a spatial smoothing on a larger window reduces the local gradients. Thus, the strain fields are globally reduced which the identification procedure interprets as a stiffer behaviour. Poisson's ratio remains unchanged as both $\varepsilon_{x x}$ and $\varepsilon_{y y}$ are affected by the smoothing (more or less in the same way).

\section{Effect of the temporal smoothing}

The influence of the temporal smoothing used to compute acceleration maps on the identified results has also been investigated. Table 6 shows the results obtained for two different kinds of reconstruction of acceleration maps: the 4th order polynomial fitting of displacement on a sliding window of nine pictures (with temporal smoothing) and the simple finite differences algorithm expressed in equation (2) (without temporal smoothing). They show a difference of $1.6 \mathrm{GPa}$ in average but the scatter is very large for the data obtained without smoothing. These results confirm the essential role of the acceleration in the proposed identification procedure and underline the necessity in this study to use temporal smoothing to get relevant acceleration maps since the measurements are too noisy to use direct finite differences.
Table 4 Identified mechanical properties-test 1 -influence of the accuracy of $a_{x}$ (obtained with VF set 1 )

\begin{tabular}{|c|c|c|c|c|c|c|c|}
\hline & \multicolumn{6}{|c|}{ Time $(\mu \mathrm{s})$} & \multirow[t]{2}{*}{ Average } \\
\hline & 6.7 & 10.0 & 13.3 & 20.0 & 23.3 & 26.7 & \\
\hline \multicolumn{8}{|c|}{ Young's modulus in $\mathrm{GPa}$ (reference value: $E=23.2 \mathrm{GPa}$ ) } \\
\hline Raw data & 20.4 & 21.1 & 15.8 & 15.7 & 16.6 & 13.6 & 17.2 \\
\hline$a_{x}+1 \cdot 10^{5} \mathrm{~m} \cdot \mathrm{s}^{-2}$ & 26.1 & 25.1 & 18.1 & 17.9 & 19.2 & 16.2 & 20.4 \\
\hline$a_{x}+2 \cdot 10^{5} \mathrm{~m} \cdot \mathrm{s}^{-2}$ & 31.8 & 29.1 & 20.5 & 20.1 & 21.7 & 18.8 & 23.7 \\
\hline \multicolumn{8}{|c|}{ Poisson's ratio (reference value: $v=0.34$ ) } \\
\hline Raw data 1 & 0.28 & 0.22 & 0.32 & 0.27 & 0.29 & 0.30 & 0.28 \\
\hline$a_{x}+1 \cdot 10^{5} \mathrm{~m} \cdot \mathrm{s}^{-2}$ & 0.28 & 0.22 & 0.31 & 0.27 & 0.29 & 0.30 & 0.28 \\
\hline$a_{x}+2 \cdot 10^{5} \mathrm{~m} \cdot \mathrm{s}^{-2}$ & 0.28 & 0.21 & 0.31 & 0.27 & 0.29 & 0.30 & 0.28 \\
\hline
\end{tabular}


Table 5 Identified mechanical properties-test 1 -influence of the smoothing

\begin{tabular}{|c|c|c|c|c|c|c|c|}
\hline & \multicolumn{6}{|c|}{ Time $(\mu \mathrm{s})$} & \multirow[t]{2}{*}{ Average } \\
\hline & 6.7 & 10.0 & 13.3 & 20.0 & 23.3 & 26.7 & \\
\hline \multicolumn{8}{|c|}{ Young's modulus in GPa (reference value: $E=23.2 \mathrm{GPa}$ ) } \\
\hline$R=10$ pitches of the grid & 20.4 & 21.1 & 15.8 & 15.7 & 16.6 & 13.6 & 17.2 \\
\hline$R=12$ pitches of the grid & 21.5 & 21.2 & 15.5 & 17.0 & 16.8 & 14.0 & 17.7 \\
\hline$R=15$ pitches of the grid & 21.5 & 21.3 & 15.3 & 18.2 & 17.5 & 14.1 & 18.0 \\
\hline$R=17$ pitches of the grid & 21.1 & 21.4 & 15.5 & 18.0 & 17.7 & 14.0 & 18.0 \\
\hline$R=20$ pitches of the grid & 21.1 & 21.5 & 16.2 & 20.1 & 18.1 & 13.9 & 18.5 \\
\hline \multicolumn{8}{|c|}{ Poisson's ratio (reference value: $v=0.34$ ) } \\
\hline$R=10$ pitches of the grid & 0.28 & 0.22 & 0.32 & 0.27 & 0.29 & 0.30 & 0.28 \\
\hline$R=12$ pitches of the grid & 0.27 & 0.21 & 0.33 & 0.28 & 0.29 & 0.30 & 0.28 \\
\hline$R=15$ pitches of the grid & 0.26 & 0.20 & 0.34 & 0.29 & 0.28 & 0.30 & 0.28 \\
\hline$R=17$ pitches of the grid & 0.25 & 0.20 & 0.34 & 0.29 & 0.28 & 0.30 & 0.28 \\
\hline$R=20$ pitches of the grid & 0.25 & 0.20 & 0.35 & 0.29 & 0.28 & 0.31 & 0.28 \\
\hline
\end{tabular}

In conclusion, even if the present results have to be improved, the fact that, in spite of the relatively low quality of the measurements, these results allow to identify mechanical properties of the right order of magnitude is very promising. As already mentioned, it has to be underlined that such a use of the VFM using only the inertial effects as information source has never been performed until now for such a high strain rate case. Assuming that improved measurements could be obtained in the future, this approach could advantageously change the testing procedures at high-strain rates. However, to be more accurate, the testing device should be designed in order to favor inertial effects. Indeed, it can be seen on the present tests that the acceleration stages are rather short in the lifespan of the specimen. A fixture ensuring inertial effects over a more significant part of the test would be very beneficial here. Since load is generally used in the standard SPHB analysis, the next section is dedicated to the analysis of the load for test 2 .

Identification of the Applied Load

\section{Using the VFM to identify the applied load}

Instead of identifying the mechanical properties from the tested material, another possibility is to identify the applied force from the measurements, assuming that the mechanical parameters are the reference ones.

By choosing a virtual field matching the condition expressed in equation (14), one has access to the resultant of the applied loading. For instance here, by choosing the following virtual field (see Fig. 20 for axes and geometry):

$$
\left\{\begin{array}{l}
U_{x}^{*}=U_{x}^{*}=0 \Rightarrow \varepsilon_{x}^{*}=\varepsilon_{y}^{*}=\varepsilon_{s}^{*}=0 \text { for } x \leq 0 \\
\left\{\begin{array}{l}
U_{x}^{*}=x \\
U_{y}^{*}=0
\end{array}\right. \\
\left\{\begin{array}{l}
U_{x}^{*}=L \\
U_{y}^{*}=0
\end{array} \Rightarrow \varepsilon_{x}^{\varepsilon_{x}^{*}=1} \begin{array}{l}
\varepsilon_{y}^{*}=0 \\
\varepsilon_{s}^{*}=0
\end{array} \quad \text { for } 0 \leq x \leq L\right.
\end{array}\right.
$$

Equation (4) becomes:

$$
Q_{x x} \int_{V} \varepsilon_{x} d V+Q_{x y} \int_{V} \varepsilon_{y} d V+\int_{V} \rho a_{x} x d V=F L .
$$

This can also be written:
$F=F_{d}+F_{i} ;$
Table 6 Identified mechanical properties-test 1 -influence of the temporal

\begin{tabular}{|c|c|c|c|c|c|c|c|}
\hline & \multicolumn{6}{|c|}{ Time $(\mu \mathrm{s})$} & \multirow[t]{2}{*}{$\overline{\text { Average }}$} \\
\hline & 6.7 & 10.0 & 13.3 & 20.0 & 23.3 & 26.7 & \\
\hline \multicolumn{8}{|c|}{ Young's modulus in GPa (reference value: $E=23.2 \mathrm{GPa}$ ) } \\
\hline Polynomial fitting & 20.4 & 21.1 & 15.8 & 15.7 & 16.6 & 13.6 & 17.2 \\
\hline Finite differences & 13.4 & 4.7 & 24.7 & 8.7 & 24.6 & 17.7 & 15.6 \\
\hline \multicolumn{8}{|c|}{ Poisson's ratio (reference value: $v=0.34$ ) } \\
\hline Polynomial fitting & 0.28 & 0.22 & 0.32 & 0.27 & 0.29 & 0.30 & 0.28 \\
\hline Finite differences & 0.27 & 0.28 & 0.31 & 0.27 & 0.29 & 0.29 & 0.29 \\
\hline
\end{tabular}
smoothing 
with:

$$
\left\{\begin{array}{l}
F_{d}=\frac{Q_{x x} \int_{V} \varepsilon_{x} d V+Q_{x y} \int_{V} \varepsilon_{y} d V}{L} \\
F_{i}=\frac{\int_{V} \rho a_{x} x d V}{L}
\end{array} .\right.
$$

$F_{d}$ is the load that is devoted to specimen deformation whereas $F_{i}$ is the inertial load needed to accelerate the specimen.

\section{Synchronization of the signals}

To be able to compare the two signals (the load identified thanks to the VFM and the transmitted load recorded thanks to the strain gauge), they have to be synchronized very carefully.

The transmitted load reaches the gauge glued onto the output bar (Fig. 1) later than it reaches the right edge of the region of interest of the sample. To know when the signal has reached this right edge, the time used by the wave to travel from it to this gauge has to be subtracted. This time is given by the following equation:

$t_{\text {travel }}=\frac{d_{\mathrm{Ti}}}{v_{\mathrm{Ti}}}+\frac{d_{\text {comp }}}{v_{\text {comp }}}$,

where $d_{\mathrm{Ti}}$ and $v_{\mathrm{Ti}}$ are respectively the distance the wave has to travel and the velocity of the wave in the titanium constituting the bars and $d_{\text {comp }}$ and $v_{\text {comp }}$ are the same parameters for the composite. Taking into account the density $\rho$ and Young's modulus $E$ of both materials, equation (28) becomes:

$t_{\text {travel }}=d_{\mathrm{Ti}} \sqrt{\frac{\rho_{\mathrm{Ti}}}{E_{\mathrm{Ti}}}}+d_{\text {comp }} \sqrt{\frac{\rho_{\text {comp }}}{E_{\text {comp }}}}$.

Using a measured value for Young's modulus of the titanium (107 GPa), a standard value for its density $\left(4,500 \mathrm{~kg} / \mathrm{m}^{3}\right)$, the reference value for Young's modulus of the composite $(23.2 \mathrm{GPa})$ and a measured value for its density $\left(2,000 \mathrm{~kg} / \mathrm{m}^{3}\right)$, and considering the measured distances on the device (see Fig. 1), this leads to:

$$
\begin{aligned}
t_{\text {travel }} & =730 \cdot 10^{-3} \sqrt{\frac{4500}{107 \cdot 10^{9}}}+85 \cdot 10^{-3} \sqrt{\frac{2000}{23.2 \cdot 10^{9}}} \\
& \simeq 175 \mu \mathrm{s} .
\end{aligned}
$$

Concerning the identified load, one has to know the exact moment when the first image was recorded. Considering the approximative time the incident tensile wave needs to reach the sample, a delay of $600 \mu \mathrm{s}$ has been imposed to trigger the camera. However, as the triggering of the camera is controlled on a per bank basis (i.e., a group of 8 physical sensors), the first recorded image is not necessarily recorded at exactly $600 \mu \mathrm{s}$ from the trigger sequence. Indeed, in the present case, the first image corresponds to the time $596 \mu$ s after the trigger signal that is to say that this value of $596 \mu \mathrm{s}$ has to be added to the temporal origin of the identified load to synchronize it with the transmitted load signal.
Fig. 21 Identified load curves compared to the load obtained from the strain gauge signal (test 2)

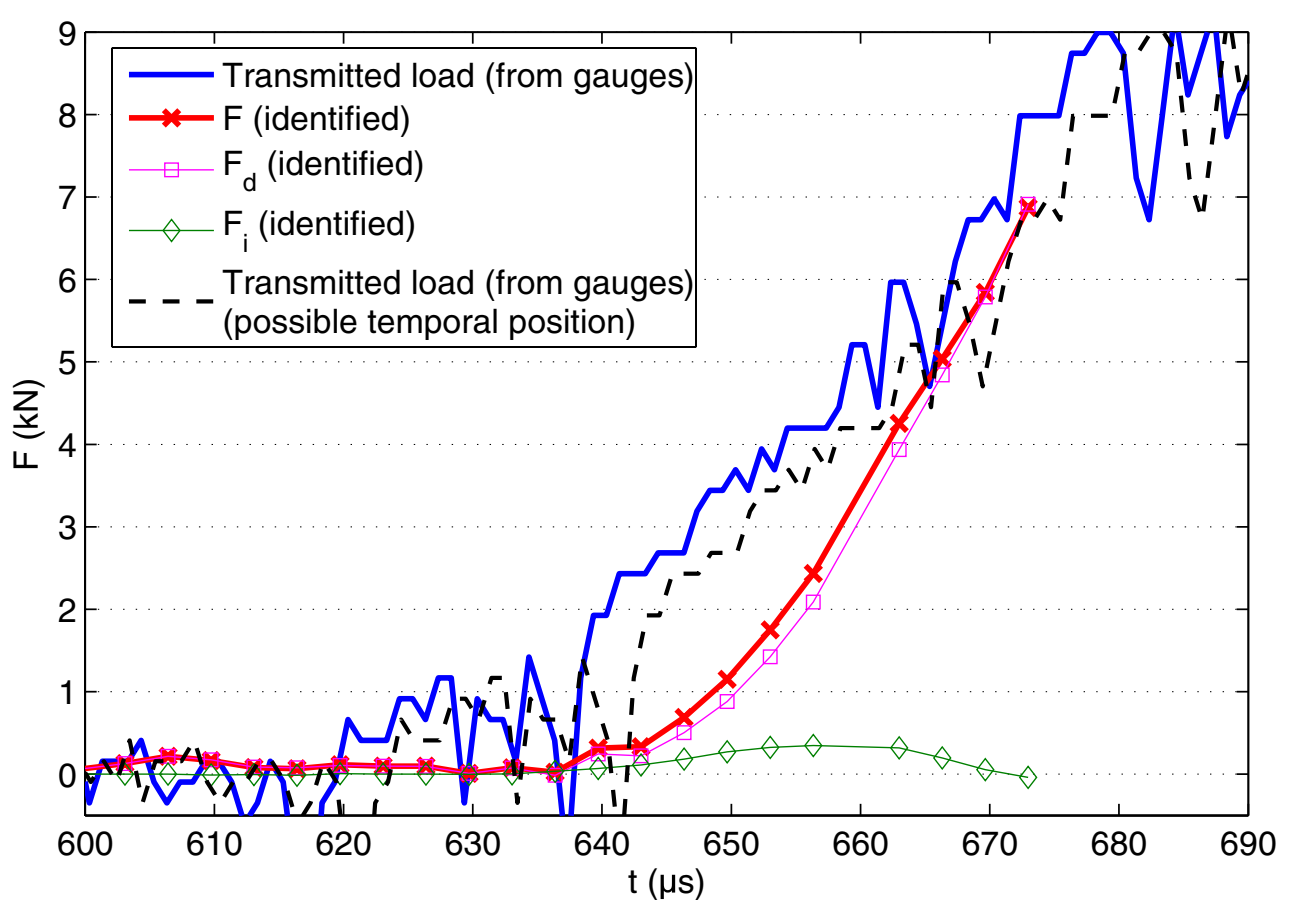




\section{Results}

By applying the approach explained above to the second test, the curves shown on Fig. 21 have been obtained (the origin of time is the moment of the physical trigger). On this figure, some temporal gap between the signals can be observed. However, this gap can be explained by the hypotheses that have been made on the value of some parameters to determine the time $t_{\text {travel }}$. Especially, Young's modulus of the composite remains an unknown: only its order of magnitude can be given. In the same way, the value of the density of the titanium is a standard value taken from the literature. By replacing the corresponding parameters by the average identified value obtained for the second test for Young's modulus of the composite $(28.5 \mathrm{GPa})$ and $4400 \mathrm{~kg} / \mathrm{m}^{3}$ for the density of the titanium, it leads to $t_{\text {travel }} \simeq 170 \mu \mathrm{s}$ (which leads to the dotted curve plotted on Fig. 21 that is better synchronized with the curves of the identified load). So, considering the uncertainty on a certain number of parameters, both signals can be considered to be reasonably synchronized.

Assuming this synchronization and considering that the gauge signal is quite noisy, the identified load and the transmitted load measured by the gauge glued onto the output bar show a global accordance over the duration of the test. The curves also show that inertial forces coming from the sample itself take part to the global forces for only a small amount because of the small and light specimen, and because of the test configuration. In another recent study based on the same principle, this term proved predominant $[49,50]$.

\section{Conclusion and Perspectives}

This paper has presented a methodology to obtain relevant full-field measurements at high strain rates using an ultra high speed camera and the grid method. Tensile quasi-isotropic glass-epoxy composite coupons with and without an open hole have been tested on a Split Hopkinson Pressure Bar fixture and the results have been processed to identify the materials elastic stiffness components. The main conclusions of this work are as follows.

- Because of the nature of the camera (multi CCD sensors and rotating mirror), it is necessary to obtain displacements by comparing the images on each sensor between a set of still images recorded before the test and that recorded during the test. Comparing images from one sensor to the next introduces parasitic displacements caused by small discrepancies in the CCDs alignment.
- The displacement resolution obtained with the grid method is about five times less with the ultra high speed camera than with a standard CCD camera.

- Strain, strain rate and acceleration maps can be obtained by smoothing and differentiating the data spatially and/or temporally. The resulting data are consistent with expectations even though the strains at the onset of cracking are very small (1\%) compared to what is obtained on metals (challenging problem).

- The data can be used to identify the stiffness components of the quasi-isotropic lay-up. The original idea of this paper is to make use of the inertial effects to identify the stiffnesses without the need of the impact force. There is a motivation to try to avoid force measurements in dynamic events because it is very difficult from strain gauge measurements, resulting in the efficient but bulky and expensive SHPB fixture. Moreover, the standard SHPB analysis relies on strong hypotheses that limits the shape of the specimen and struggles with the initial part of the test because of inertia effects. This early stage is however where the elastic stiffnesses have to be identified, hence the difficulties usually encountered to do this.

- The Virtual Fields Method has been used successfully to extract the composites stiffness components. Both tests yielded about the same value for both Young's modulus and Poisson's ratio. A sensitivity analysis to smoothing, noise magnitude and choice of the virtual fields resulted in rather stable identified values which gives confidence in the results.

- Finally, the stiffness data were used to reconstruct the load using the Virtual Fields Method and the results have been compared to the load obtained from the strain gauges on the output bar. The results compare favorably all over the duration of the test (considering the high level of noise on the gauge signal).

Clearly, this paper is seminal in nature and does not claim to present a ready-to-use procedure. Its objective is to attract the attention of the high strain rate testing community to the fact that when full-field measurements are performed, there are alternative ways of processing the data by making use of inertial forces that are directly measured onto the specimen. As a consequence, this opens up a wide area of future research where new tests could be designed where inertial effects could be made predominant and no impact force would be necessary. If the ultra high strain rate cameras are still expensive and wanting in image quality for 
measurement purposes, there is no doubt that the technology will improve in the future and that the present type of procedure could become a real alternative to the present tests. Among the problems that could be addressed are:

- Heterogeneous materials (like welds for instance) where spatially varying mechanical properties could be obtained with the same approach as that developed in [51].

- Identification of a strain rate dependance law over a range of strain rates with a single specimen making use of the presence of an heterogeneous strain rate map, as in [6].

- Explore the identification of more complex elastoplastic models (including cyclic loads) by using heterogeneous tests as in $[52,53]$.

The main tracks to be followed in the future to make progress on the type of procedure presented here is the improvement of the quality of the measurement and the the design of suitable test configurations. While the first one is largely dependent on the quality of the cameras, the second point will require significant effort in the future where numerical simulations will have to be employed to validate the designs with respect to the materials parameters to be identified. Some exploratory work is underway on metals [50].

Acknowledgements The authors would like to express their gratitude to the UK Engineering and Physical Sciences Research Council for their financial support to this project (Grant $n^{\circ}$ : EP/G001715/1) and the loan of the Ultra High Speed camera through the Engineering Instrument Pool.

\section{References}

1. Tiwari V, Sutton MA, McNeill SR, Xu S, Deng X, Fourney WL, Bretall D (2009) Application of 3D image correlation for full-field transient plate deformation measurements during blast loading. Int J Impact Eng 36:862-874

2. Reu PL, Miller TJ (2008) The application of high-speed digital image correlation. J Strain Anal 43:673-688

3. Tarigopula V, Hopperstad OS, Langseth M, Clausen AH, Hild F (2008) A study of localisation in dual-phase highstrength steels under dynamic loading using digital image correlation and FE analysis. Int J Solids Struct 45:601-619

4. Schmidt T, Gilat A (2008) 3D image correlation studies of geometry and materiakl property effects during split Hopkinson bar experiments. In: Proceedings of the XIth international congress and exposition on experimental and applied mechanics

5. Kajberg J, Wikman B (2007) Viscoplastic parameter estimation by high strain-rate experiments and inverse modellingspeckle measurements and high-speed photography. Int $\mathrm{J}$ Solids Struct 47:145-164

6. Avril S, Pierron F, Sutton MA, Yan J (2008) Identification of elasto-visco-plastic parameters and characterization of
Lüders behavior using digital image correlation and the virtual fields method. Mech Mater 40:729-742

7. Tiwari V, Sutton MA, McNeill SR (2007) Assessment of high speed imaging systems for 2D and 3D deformation measurements: methodology development and validation. Exp Mech 47:561-579

8. Honour J (2009) A brief history of principles used in high speed cameras. Imaging Sci J 57:303-316

9. Luo H, Lu H, Leventis N (2006) The compressive behavior of isocyanate-crosslinked silica aerogel at high strain rates. Mech Time-Depend Mater 10:83-111

10. Luo H, Churu G, Fabrizio EF, Schnobrich J, Hobbs A, Dass A, Mulik S, Zhang Y, Grady BP, Capecelatro A, SotiriouLeventis C, Leventis N (2008) Synthesis and characterization of the physical, chemical and mechanical properties of isocyanate-crosslinked vanadia aerogels. J Sol-Gel Sci Technol 48:113-134

11. Siviour CR (2009) A measurement of wave propagation in the split Hopkinson pressure bar. Meas Sci Technol 20:065702 (5 pp)

12. Kirugulige MS, Tippur HV (2009) Measurement of fracture parameters for a mixed-mode crack driven by stress waves using image correlation technique and high-speed digital photography. Strain 45:108-122

13. Harding J, Welsh LM (1983) A tensile testing technique for fiber-reinforced composites at impact rates of strain. J Mater Sci 18:1810-1826

14. Harding J, Wood EO, Campbell JD (1960) Testing of materials at impact rates of strain. J Mech Eng Sci 2:88-96

15. Staab GH, Gilat A (1995) High-strain rate response of angle-ply glass epoxy laminates. J Compos Mater 29:13081320

16. Staab GH, Gilat A (1991) A direct-tension split Hopkinson bar for high strain-rate testing. Exp Mech 31:232-235

17. Li M, Wang R, Han M-B (1993) A Kolsky bar: tension, tension-tension. Exp Mech 33:7-14

18. Hamouda AMS, Hashmi MSJ (1998) Testing of composite materials at high rates of strain: advances and challenges. J Mater Process Technol 77:327-336

19. Sierakowski RL (1997) Strain rate effects in composites. Appl Mech Rev 50:741-762

20. Barre S, Chotard T, Benzeggagh ML (1996) Comparative study of strain rate effects on mechanical properties of glass fibre-reinforced thermoset matrix composites. Composites A 27:1169-1181

21. Shokrieh MM, Omidi MJ (2009) Tension behavior of unidirectional glass/epoxy composites under different strain rates. Compos Struct 88:595-601

22. Goldberg RK, Gilat A (2002) Experimental and computational characterization of the high strain rate tensile response of polymer matrix composites. In: 14 th symposium on composite materials. Pittsburgh, PA

23. Medina JL, Harding J (2000) The effect of strain rate on the through-thickness tensile stiffness and strength properties of fibre-reinforced epoxy composites. In: 6th international conference on mechanical and physical behaviour of materials under dynamic loading. Krakow, Poland

24. Awerbuch J, Madhukar MS (1985) Notched strength of composite laminates: predictions and experiments-a review. J Reinf Plast Compos 4:3-159

25. Kazemahvazi S, Zenkert D, Burman M (2009) Notch and strain rate sensitivity of non-crimp fabric composites. Compos Sci Technol 69:793-800

26. Chen Y, Hallett SR (2008) High-strain rate effects on open hole tensile strength of composites. In: 4th international conference on composites testing and model 
identification (CompTest). Dayton. academic.udayton.edu/ stevendonaldson/comptest2008.html

27. Field JE, Walley SM, Proud WG, Goldrein HT, Siviour CR (2004) Review of experimental techniques for high rate deformation and shock studies. Int J Impact Eng 30:725-775

28. Piro J-L, Grédiac M (2004) Producing and transferring lowspatial-frequency grids for measuring displacement fields with the moiré and grid methods. Exp Tech 28:23-26

29. Surrel Y (1994) Moiré and grid methods: a signal-processing approach. In: Pryputniewicz RJ, Stupnicki J (eds) Interferometry '94: photomechanics, vol 2342. SPIE, Bellingham

30. Moulart R, Rotinat R, Pierron F, Lerondel G (2007) On the realization of microscopic grids for local strain measurement by direct interferometric photolithography. Opt Lasers Eng 45:1131-1147

31. Finston MI (1985) Fundamentals of optical design in rotating mirror cameras. Opt Laser Technol 17:83-88

32. Kirugulige MS, Tippur HV, Denney TS (2007) Measurement of transient deformations using digital image correlation method and high-speed photography: application to dynamic fracture. Appl Opt 46:5083-5096

33. Moulart R, Avril S, Pierron F (2006) Identification of the through-thickness rigidities of a thick laminated composite tube. Composites A 37:326-336

34. Pierron F, Green B, Wisnom MR (2007) Full-field assessment of the damage process of laminated composite open-hole tensile specimens. Part I: methodology. Composites A 38:23072320

35. Bioucas-Dias J, Valadão G (2007) Phase unwrapping via graph cuts. IEEE Trans Image Process 16:698-709

36. Cleveland W, Loader C (1996) Smoothing by local regression: principles and methods. In: Haerdle W, Schimek MG (eds) Statistical theory and computational aspects of smoothing. Springer, Berlin, pp 10-49

37. Avril S, Feissel P, Pierron F, Villon P (2008) Estimation of the strain field from full-field displacement noisy data: comparing finite elements global least squares and polynomial diffuse approximation. Eur J Comput Mech 17:857-868

38. Pierron F, Green B, Wisnom MR, Hallett SR (2007) Fullfield assessment of the damage process of laminated composite open-hole tensile specimens. Part I: experimental results. Composites A 38:2321-2332

39. Grédiac M (1989) Principe des travaux virtuels et identification. C R Acad Sci 309:1-5 (In French with abridged English version)

40. Grédiac M, Pierron F, Avril S, Toussaint E (2006) The virtual fields method for extracting constitutive parameters from full-field measurements: a review. Strain (An International Journal for Experimental Mechanics) 42:233-253
41. Grédiac M, Paris P-A (1996) Direct identification of elastic constants of anisotropic plates by modal analysis: theoretical and numerical aspects. J Sound Vib 195:401-415

42. Grédiac M, Fournier N, Paris P-A, Surrel Y (1998) Direct identification of elastic constants of anisotropic plates by modal analysis: experimental results. J Sound Vib 210:643659

43. Giraudeau A, Pierron F (2005) Identification of stiffness and damping properties of thin isotropic vibrating plates using the virtual fields method. Theory and simulations. J Sound Vib 284:757-781

44. Giraudeau A, Guo B, Pierron F (2006) Stiffness and damping identification from full field measurements on vibrating plates. Exp Mech 46:777-787

45. Giraudeau A, Pierron F, Guo B (2010) An alternative to modal analysis for material stiffness and damping identification from vibrating plates. J Sound Vib 329:16531672

46. Grédiac M, Toussaint E, Pierron F (2002) Special virtual fields for the direct determination of material parameters with the virtual fields method. 1 - principle and definition. Int J Solids Struct 39:2691-2705

47. Grédiac M, Toussaint E, Pierron F (2002) Special virtual fields for the direct determination of material parameters with the virtual fields method. 2-application to in-plane properties. Int J Solids Struct 39:2706-2725

48. Cui WC, Wisnom MR (1992) Contact finite element analysis of three- and four-point short-beam bending of unidirectional composites. Compos Sci Technol 45:323-334

49. Pierron F, Sutton MA, Tiwari V (2010) Ultra high speed DIC on a three point bending test mounted on a Hopkinson bar. In: 2010 SEM annual conference and exposition on experimental and applied mechanics. Indianapolis, USA

50. Pierron F, Sutton MA, Tiwari V (2010) Ultra high speed DIC and Virtual Fields Method analysis of a three point bending impact test on an aluminium bar. Exp Mech. doi:10.1007/s11340-010-9402-y

51. Sutton MA, Yan J, Avril S, Pierron F, Adeeb S (2008) Identification of heterogeneous constitutive parameters in a welded specimen: uniform stress and virtual fields methods for material property estimation. Exp Mech 48:451-464

52. Cooreman S, Lecompte D, Sol H, Vantomme J, Debruyne D (2008) Identification of mechanical material behaviour through inverse modeling and DIC. Exp Mech 48:421-433

53. Avril S, Pierron F, Pannier Y, Rotinat R (2008) Stress reconstruction and constitutive parameter identification in elastoplasticity using measurements of deformation fields. Exp Mech 48:403-419 